University of Rhode Island

DigitalCommons@URI

\title{
Volcanic Rocks in the Narragansett Basin, Southeastern New England: Petrology and Significance to Early Basin Formation
}

Anton Maria

University of Rhode Island

O. Don Hermes

University of Rhode Island, dhermes@uri.edu

Follow this and additional works at: https://digitalcommons.uri.edu/geo_facpubs

Terms of Use

All rights reserved under copyright.

\section{Citation/Publisher Attribution}

Maria, A., \& Hermes, O. D. (2001). Volcanic Rocks in the Narragansett Basin, Southeastern New England:

Petrology and Significance to Early Basin Formation. AJS Online, 301(3), 286-312. doi: 10.2475/

ajs.301.3.286

Available at: http://dx.doi.org/10.2475/ajs.301.3.286

This Article is brought to you for free and open access by the Geosciences at DigitalCommons@URI. It has been accepted for inclusion in Geosciences Faculty Publications by an authorized administrator of DigitalCommons@URI.For more information, please contact digitalcommons-group@uri.edu. 


\title{
VOLCANIC ROCKS IN THE NARRAGANSETT BASIN, SOUTHEASTERN NEW ENGLAND: PETROLOGY AND SIGNIFICANGE TO EARLY BASIN FORMATION
}

\author{
ANTON MARIA* and O. DON HERMES
}

Department of Geosciences, University of Rhode Island, Kingston, Rhode Island, 02881

\begin{abstract}
A suite of volcanic rocks in the northwest corner of the Narragansett basin includes at least four basalt flows, two rhyolite flows, and associated pyroclastic rocks. The volcanics are interbedded with non-marine, sedimentary rocks within the lower part of the Wamsutta Formation and, until recently, were considered Pennsylvanian in age. An age of about $373 \mathrm{Ma}$ has been reported for the rhyolite, based on $\mathrm{U} / \mathrm{Pb}$ geochronology (Thompson and others, 1999). The basalt flows are typically 1 to $2 \mathrm{~m}$ thick and marked by pillows, sediment dikes, and magma/sediment commingling features. The two younger flows contain intergranular to subophitic clinopyroxene and sparse phenocrysts of plagioclase and pseudomorphed olivine in a pilotaxitic groundmass. The two older flows are similar but contain no pyroxene. The rhyolite flows, from 3 to $20 \mathrm{~m}$ thick, are characterized by subhorizontal quartz seams that represent cooling cracks or zones of preferential vesiculation filled with late-stage quartz. Both rhyolite flows contain phenocrysts of anorthoclase in a granophyric, devitrified groundmass with relict perlitic features. Identification of pyroclastic deposits beneath one rhyolite flow indicates that extrusion was preceded by explosive activity. Major and trace elements indicate that the volcanics are mildly alkaline, and geochemical trends suggest the basalt and rhyolite originated by partial melting of different sources, followed by limited fractional crystallization. Apparent restriction to early basin sediments suggests that the volcanics reflect a rifting event associated with the formation of the Narragansett basin. Though it is now apparent that the basin was active well before the Pennsylvanian, it is not clear whether the rift event was related to the extensional environment that prevailed through much of the Paleozoic, producing the alkalic plutonism prominent in this part of the Avalon Zone. Similarities to Devonian-Carboniferous bimodal suites in the Maritimes basin of Canada suggest possibly analogous origins.
\end{abstract}

INTRODUCTION

A suite of volcanic rocks is located in South Attleboro, Massachusetts, within the northwest corner of the Narragansett basin. These rocks are the only volcanics within the Narragansett basin, aside from two isolated Precambrian units along the northern and northeastern margins of the basin (Zen, 1983). Although the South Attleboro volcanics were noted almost a century ago (Shaler and others, 1899), they were never mapped in detail. Since then, the number of units, their compositional characteristics, and even their designation as sills, dikes, or lava flows have been matters of controversy (Eaton, 1925; Cleaves, ms; Lytton, ms; Woods, ms).

As a result of the present work, it is clear that the South Attleboro suite is composed of mafic and felsic lava flows as well as associated pyroclastic units. The bimodal assemblage of basalt and rhyolite is locally distinct, despite the wide variety of igneous rocks in southeastern New England. Mafic and felsic volcanics are also found just north of the Narragansett basin, within the Norfolk basin of Massachusetts. On the geologic map of Massachusetts (Zen, 1983), the Norfolk basin volcanics and the South Attleboro volcanics are both designated by the name Wamsutta volcanics, due to their association with the Wamsutta Formation. However, this paper does not address 02882

*Present address: Graduate School of Oceanography, University of Rhode Island, Narragansett, RI 
Norfolk basin rocks, and the name Wamsutta volcanics here refers specifically to the South Attleboro suite.

A primary goal of this study is to characterize the Wamsutta volcanics so that their place within the prolonged and varied geologic history of southeastern New England may be determined. The petrologic characteristics of this bimodal volcanic suite are typical of extensional, rift volcanism (Noble, 1972; Baker and others, 1977; Barberi and others, 1982) and shed light on the tectonic history of the region's Avalon terrane. The significance of the volcanics is increased by the fact that they represent the only known occurrence of such volcanism in southeastern New England. The Wamsutta volcanics reinforce evidence from plutonic rocks indicating that a long period of episodic extensional tectonics prevailed in southeastern New England throughout much of the Paleozoic (Hermes and Zartman, 1992) and may possibly represent a culmination of this period. In addition, because the volcanic rocks appear to be contemporaneous with the older sedimentary rocks of the Narragansett basin, it is likely that their origin is related to the rift-tectonics associated with the formation of the basin. Information regarding the timing of basin formation has already been provided by radiometric dating of the Wamsutta rhyolite (Thompson and others, 1999). However, the reported age of about $373 \mathrm{Ma}$ is older than what has been accepted for rocks in the Narragansett basin, raising new questions regarding the basin's history.

GEOLOGIC SETTING

The Avalon Zone of southeastern New England forms the eastern flank of the Appalachians and contains a diverse assortment of igneous rocks ranging in age from late Proterozoic to Permian. The Narragansett basin lies east of the Hope Valley Shear Zone (fig. 1), within the Esmond-Dedham subterrane (O'Hara and Gromet, 1985; Hermes and others, 1994). Though the subterrane is dominated by plutonic rocks, volcanic rocks are present, frequently associated with the plutonic suites.

Rocks of the Esmond-Dedham subterrane record a varied tectonic history. During the late Proterozoic, calcalkaline rocks were produced during a period of plate convergence, crustal thickening, and continental accretion (Hermes and Murray, 1988; Hermes and others, 1994). The Mattapan and Lynn Volcanics, associated with the Dedham Plutonic suite, are representative of this Late Proterozoic, calcalkaline phase (Thompson and Hermes, 1990; Hermes and Murray, 1990). Throughout much of the Paleozoic, alkaline and peralkaline plutons and volcanic rocks were generated episodically, indicating an extensional tectonic environment (Hermes and Zartman, 1992 and references therein). The Spencer Hill Volcanics, associated with the Devonian Scituate Plutonic suite, are examples of this alkaline magmatism (Hermes and Murray, 1990). The Wamsutta volcanics appear to represent the final event of this long, episodic extensional phase. A switch from extensional to compressional tectonics in the Permian, during the waning stages of the Alleghanian orogeny, resulted in the production of peraluminous to metaluminous, S-type granites (Zartman and Hermes, 1987).

The Narragansett basin consists primarily of nonmarine, clastic sedimentary rocks of the Narragansett Bay Group (Hermes and others, 1994), which rest unconformably upon Late Proterozoic basement of the Esmond-Dedham subterrane. The Wamsutta Formation, which overlies the basal Pondville Conglomerate, is the only member of the Narragansett Bay Group that contains volcanic rocks. The Wamsutta Formation is composed primarily of nonmarine conglomerates and fine-grained clastic rocks (Mutch, 1968; Lidback, ms; Severson, ms; Cazier, ms) deposited as fluvial channel, point-bar, and floodplain sediments and has an estimated thickness of $595 \mathrm{~m}$ (Skehan and others, 1986).

Deformation of the metasedimentary rocks of the Narragansett basin is attributed to the Alleghanian orogeny (Cogswell and Mosher, 1994). In the southern part of the 


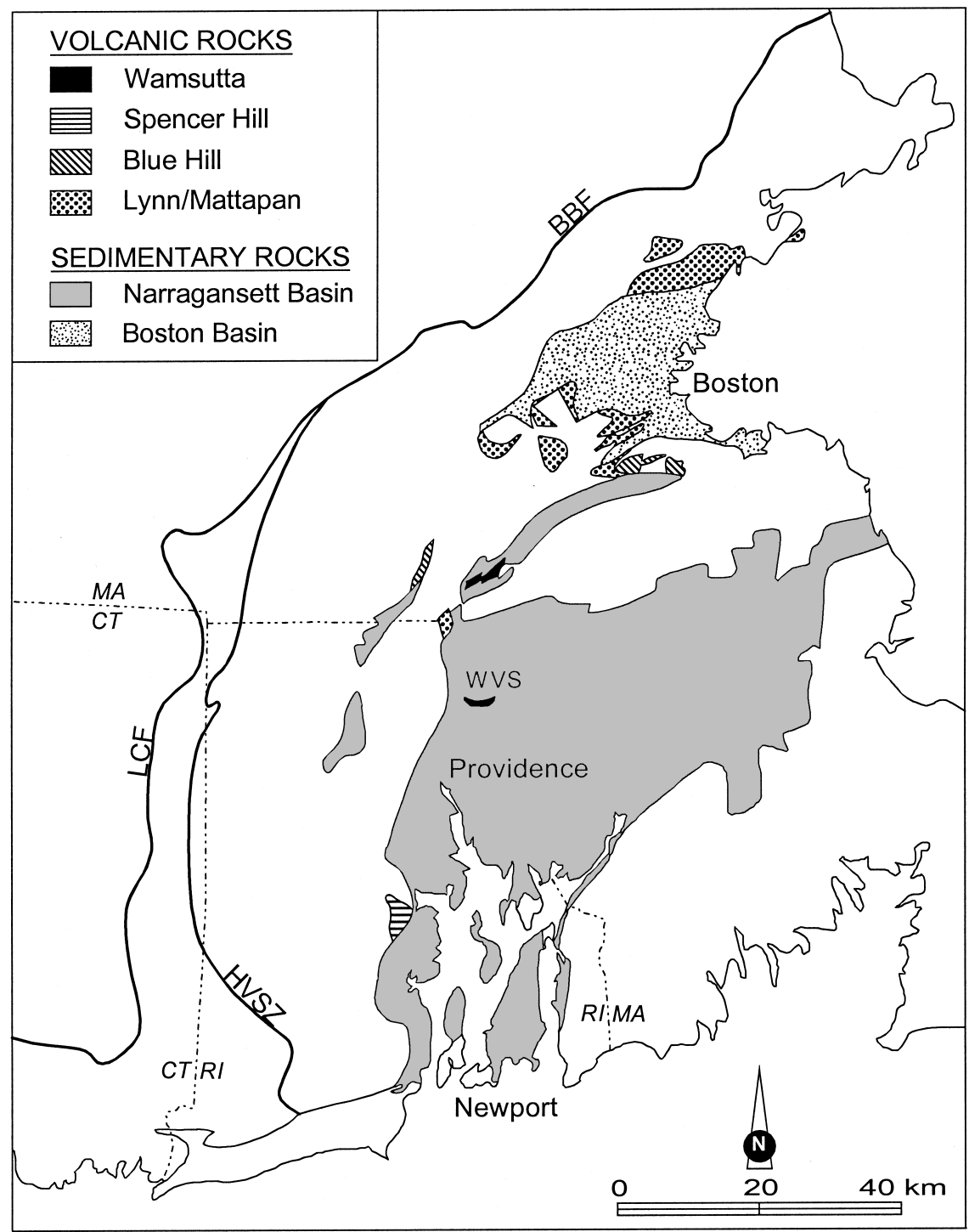

Fig. 1. Generalized geologic map of the southeastern New England Avalon Zone, showing the Narragansett basin and the location of the Wamsutta volcanic suite (WVS). Also shown are the Bloody Bluff Fault (BBF), the Lake Char Fault (LCF), and the Hope Valley Shear Zone (HVSZ).

basin, the rocks were polydeformed and metamorphosed to sillimanite grade (Grew and Day, 1972; Murray and Skehan, 1979; Mosher, 1981, 1983; Murray, 1988; Cogswell and Mosher, 1994). Effects of the orogeny were weaker in northern parts of the basin. In the vicinity of the volcanic rocks, the Wamsutta Formation was folded (Attleboro syncline), foliated, and weakly metamorphosed to anchizone grade (Hepburn and Rehmer, 1981), corresponding to zeolite to lower-greenschist facies. Subsequent brittle deformation produced high-angle, normal, and strike-slip faults (Skehan and Murray, 1979). Though some of the volcanic rocks exhibit effects of deuteric alteration, there is no indication of metamorphism. 
Age of the Wamsutta volcanics.-The age of the Narragansett basin has long been accepted as Middle to Late Pennsylvanian. A Carboniferous age is based primarily on plant fossils found in the Rhode Island Formation, which overlies the Wamsutta Formation (Skehan and others, 1979, 1986; Murray and others, 1981). The age of the Wamsutta Formation is interpreted to be Westphalian C (Lyons, ms and 1984; Lyons and Chase, 1976; Lyons and Darrah, 1977), based on fossils in the upper part of the formation (Knox, 1944). However, there is apparently no fossil control on the age of the lower portion of the Wamsutta Formation, which contains the volcanics, or on the age of the underlying basal Pondville Conglomerate. Reported fossils in the Pondville are actually located in the Norfolk basin (Lyons and others, 1976). A Late Devonian age of about $373 \mathrm{Ma}$ was recently reported for the upper rhyolite flow of the Wamsutta volcanic suite (Thompson and others, 1999). This result is the weighted mean ${ }^{207} \mathrm{~Pb} /{ }^{206} \mathrm{~Pb}$ age of three zircon fractions, two of which yielded concordant analyses. This is the first direct evidence of rocks older than Pennsylvanian within the Narragansett basin and indicates that the basin records a lengthier history than previously believed. The 60 my discrepancy between the dated rhyolite and the overlying fossiliferous Wamsutta Formation suggests an intervening unconformity, or possibly unrecognized faults.

\section{FIELD RELATIONSHIPS}

The geologic map of the study area (fig. 2) shows a conformable, synclinally folded sequence of basaltic and rhyolitic flows interstratified with clastic sedimentary rocks of the Wamsutta Formation. The generally lenticular volcanic units either pinch out along strike or are truncated by northeast-trending, high-angle faults. Nevertheless, a coherent stratigraphy is preserved on a local scale that indicates the presence of at least two discrete rhyolite layers and four basaltic units. The basalt flows extend along a chain of outcrops that is approx $9 \mathrm{~km}$ long; the rhyolite flows extend approx $5.5 \mathrm{~km}$. Two rhyolitic dikes north of I-295 (fig. 2, points 1 and 2) exhibit pronounced flow bands and may represent relict feeders to surface lava flows. Pyroclastic deposits were discovered beneath the younger rhyolite flow. However, because the pyroclastic rocks are difficult to identify without the aid of thin sections, their full extent has not yet been determined.

In most locations the lava flows are separated by relatively thin horizons of sedimentary rock. Clasts of basalt and rhyolite in sections of interbedded conglomerate attest to the intimate association between the sedimentary and volcanic rocks. However, direct contacts between lava flow units are exposed at some locations. At point 7 (fig. 2), a basalt flow directly overlies another, with a vesiculated horizon near the middle of the outcrop marking the top of the lower flow. Farther to the south, the flows separate, perhaps due to undulating topography on the original surface of deposition. Basalt flows at location 8 appear to be similarly juxtaposed. At location 6 , rhyolite is found in immediate contact with basalt.

The basalt flows are typically 1 to $2 \mathrm{~m}$ thick (locally reaching up to $6 \mathrm{~m}$ ) and show little evidence of erosion, suggesting rapid burial. Bases of flows exhibit commingling with unconsolidated sediment, and sedimentary dikes, a few to tens of centimeters thick, commonly cut the basalts, suggesting simultaneous sedimentation and volcanism. Where visible, flow laminae in the sediment dikes are consistent with injection from below. Upper contacts of basalt flows do not show sediment interaction features but are typically highly vesicular. Pillow structures are exhibited locally. All these traits indicate that the basalt units are flows and are inconsistent with earlier characterization as sills (Shaler and others, 1899; Cleaves, ms; Lytton, ms).

Like the basalts, the rhyolite flows are generally well preserved. However, the uneven outcrop pattern of the capping rhyolite may be partly due to erosion, as there are abundant rhyolite clasts in the overlying conglomerate. The upper contact of this 


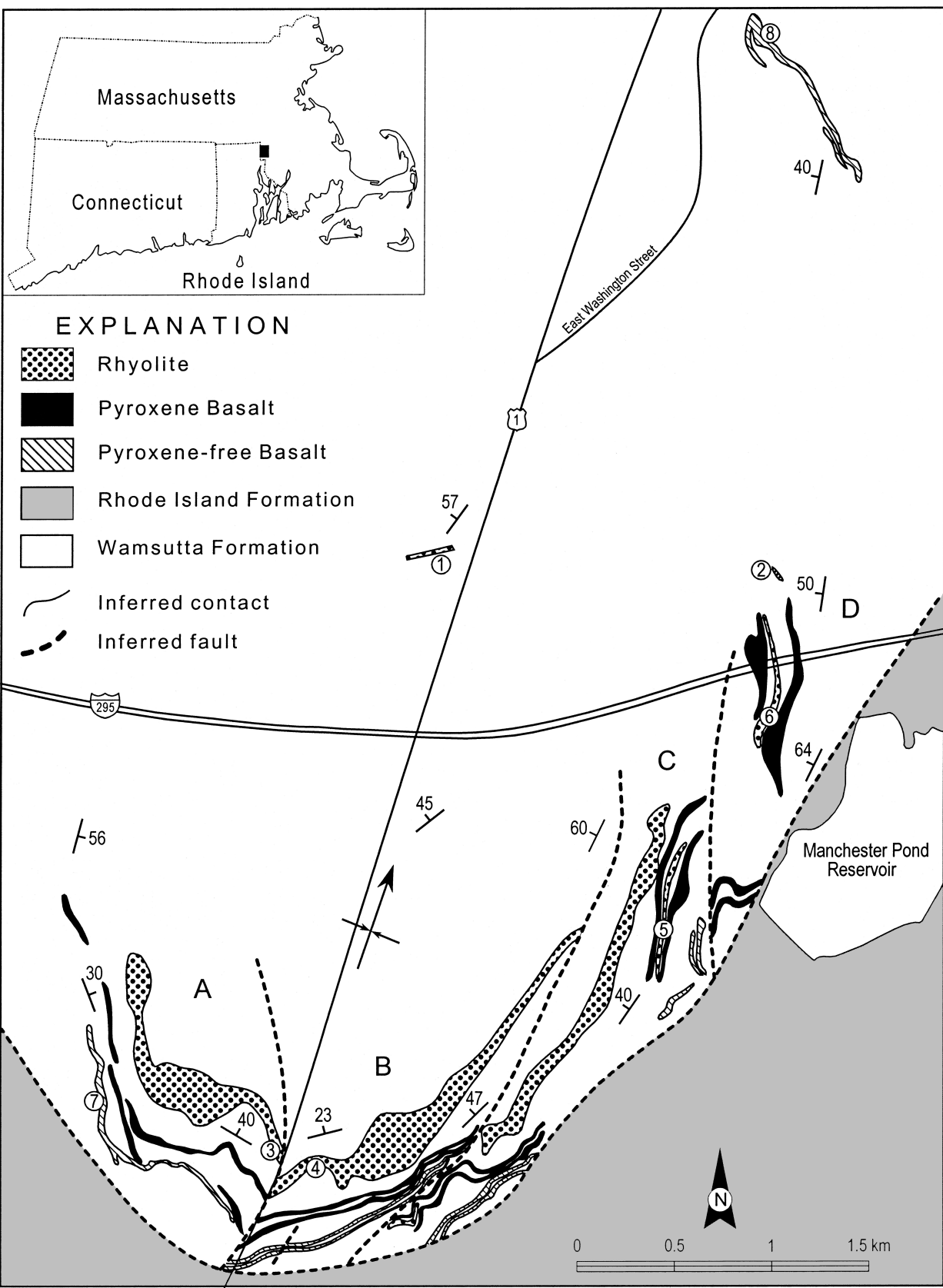

Fig. 2. Geologic map of the South Attleboro, Massachusetts, area, showing the Wamsutta volcanics interstratified with the Wamsutta Formation. Fault-bounded blocks are labeled A, B, C, and D, from west to east. In blocks A, B, and C, a relatively thick rhyolite flow tops the volcanic sequence. This capping rhyolite is underlain by four, discrete, thin flows of basalt in blocks B and C. In block A, the capping rhyolite is also underlain by four basalt flows, but the two oldest basalts are superimposed. In blocks $\mathrm{C}$ and $\mathrm{D}$, a second, thinner rhyolite flow is sandwiched between two basaltic layers. Numbers 1 through 8 indicate locations described in the text. 
rhyolite flow is not exposed, but the unit is separated from overlying sedimentary rocks by only 7 to $8 \mathrm{~m}$. The bottom contact of this flow is exposed at points 3 and 4 (fig. 2). The maximum thickness of the capping rhyolite is estimated to be $20 \mathrm{~m}$. Thickness of the lower rhyolite flow is constrained to approx $3 \mathrm{~m}$ by two enclosing basalt layers as well as exposed basal contacts at points 5 and 6 (fig. 2).

Both rhyolite flows are pink to red in color and are characterized by abundant sub-horizontal quartz seams (thin layers of quartz $\sim 1 \mathrm{~mm}$ thick) and strings of feldspar phenocrysts. Woods (ms and 1961) described these features as flow-banding, with layers of quartz and feldspar representing "relic zones of chemical heterogeneity within the magma that have been drawn out by conditions of flowage." Though the orientation of the seams and strings might be related to flow, we interpret the seams to represent either zones of preferential vesiculation or cooling cracks, filled with late-stage quartz. The seams are most abundant and closely spaced ( $5 \mathrm{~mm}$ apart) just above the basal zone. Near the top of the flow, nearly spherical, quartz-filled vesicles are more prominent. The lowermost zone (1-2 m.) of both rhyolite flows (exposed at points 3 and 5, respectively) is distinguished by a greenish-gray color and greater abundance of feldspar phenocrysts, strongly aligned in a flow-banded matrix, and may represent basal vitrophyre (now devitrified). At some locations (points 4 and 6), basal sections are the usual pink color and do not appear to be vitrophyric.

Cleaves (ms and 1929) described the rhyolite as intrusive; Shaler and others (1899), Eaton (1925), and Woods (ms and 1961) concluded that the rhyolite is extrusive; Lytton ( $\mathrm{ms}$ and 1941) was undecided on this question. The absence of exposed upper contacts makes this determination difficult. However, uniform variation in the quantity of quartz-filled seams and vesicles from bottom to top (Woods, ms), the presence of basal vitrophyre, and perlitic textures suggest that the rhyolite units are not sills. The absence of pumice fragments, lithic fragments, or other pyroclastic features within the rhyolite and the uniform, intact nature of phenocrysts suggest that the rhyolite is a lava flow rather than a pyroclastic flow. The presence of flow banding and textures associated with quenching and devitrification strengthen this conclusion.

Verification of the presence of pyroclastic deposits in association with the Wamsutta volcanics is an important outcome of our field study. Eaton (1925) reported that the basalts are associated with volcanic ash, and Woods (ms and 1961) suggested that sedimentary units beneath the rhyolite may contain ash, but neither supported these statements with details. Petrographic analysis of fine-grained rocks which we collected from beneath the youngest rhyolite flow (fig. 2, point 3) shows that they are pyroclastic. The stratigraphy for 2 to $3 \mathrm{~m}$ below the rhyolite flow at this location consists mainly of fine- and medium- to coarse-grained tuff. These pyroclastic rocks demonstrate that the younger rhyolite flow was preceded by an explosive eruptive stage. It is likely that pyroclastic and epiclastic deposits of volcanic material may be associated with the other flows as well and may constitute a significant component of the Wamsutta stratigraphy.

\section{PETROGRAPHY}

The mafic volcanics of the Wamsutta volcanic suite fall into two types, distinguished by the presence or absence of groundmass clinopyroxene. The two younger flows contain variable amounts (5-25 percent) of pale brown, intergranular to subophitic, titanium-rich augite (Ca: $\mathrm{Mg}: \mathrm{Fe}=42: 40: 18$; see Maria, ms). The younger flows also have a slightly lower abundance of opaque minerals than the older flows. Both basalt types contain sparse plagioclase phenocrysts and pseudomorphs of euhedral olivine microphenocrysts in a pilotaxitic groundmass of plagioclase, opaque minerals (predominantly ilmenite), and apatite (fig. 3A). Rapid cooling is suggested by scattered radial growths (open spherulites) of slender plagioclase crystals and occasional feathered edges on crystals. 


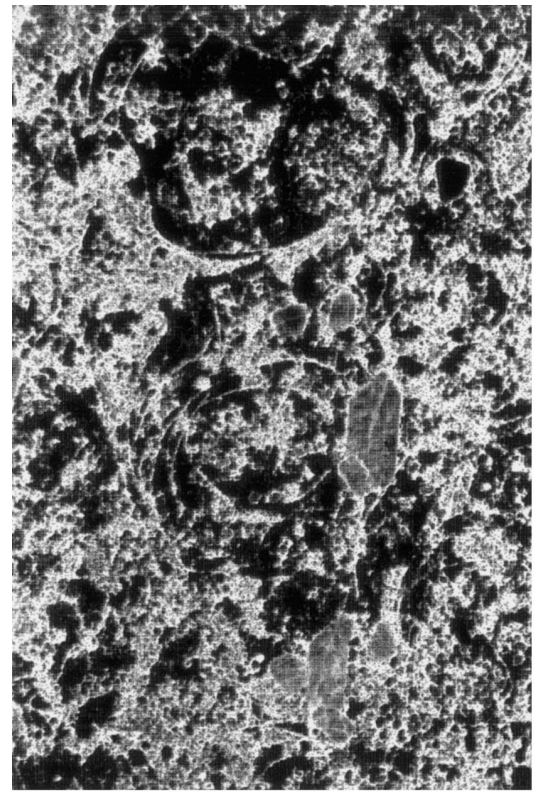

ص

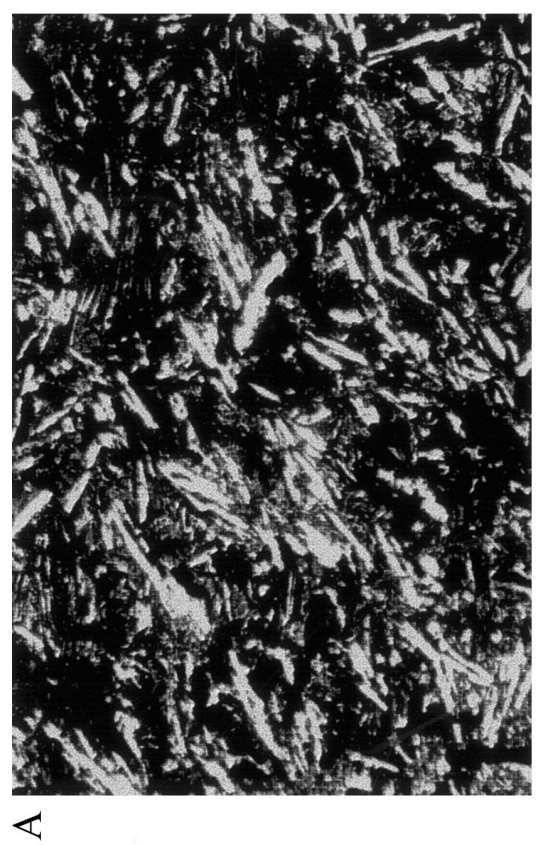

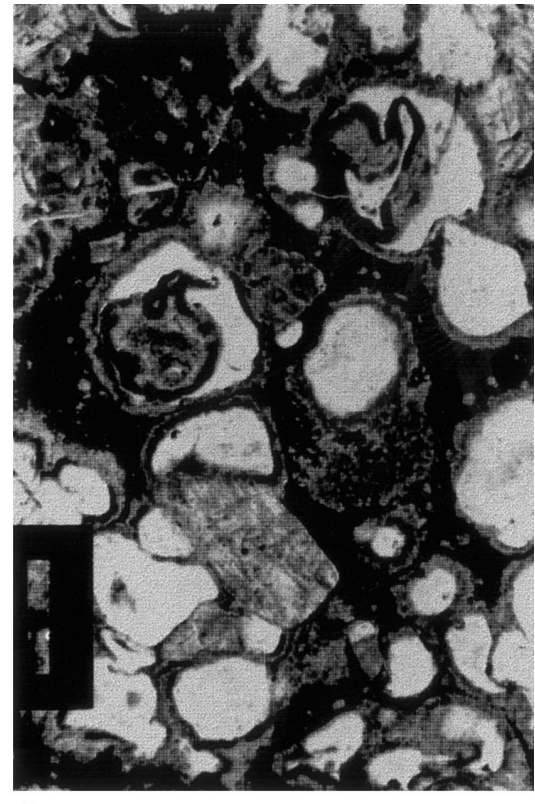

$\triangle$

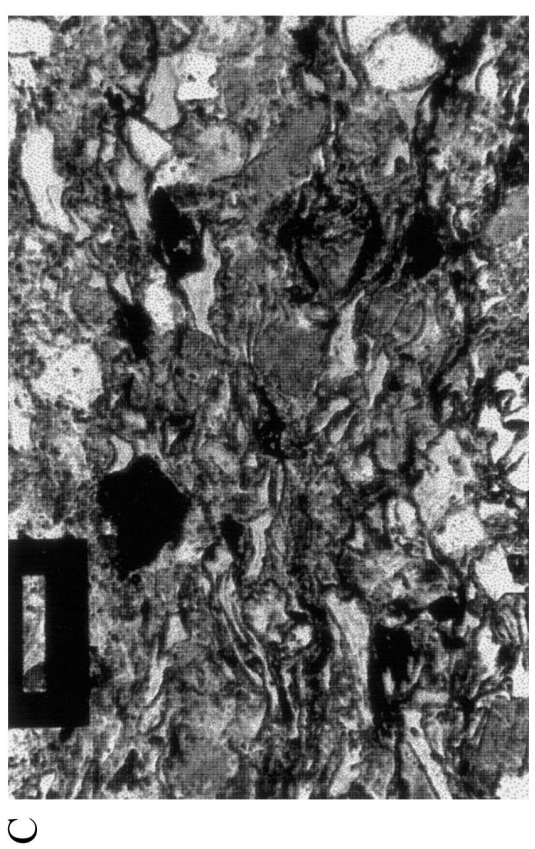

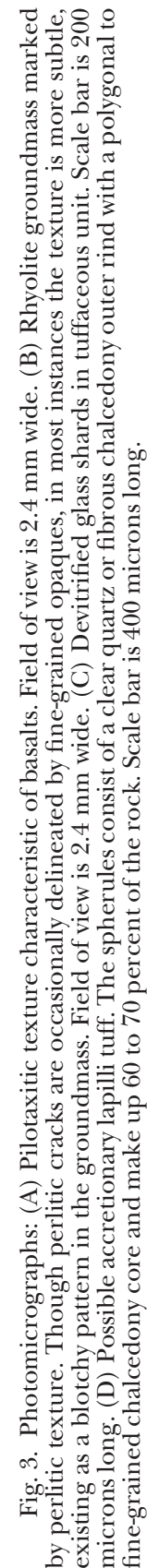


Widespread replacement of primary minerals by calcite, chlorite, epidote, granular sphene, and clay minerals indicates that the basaltic rocks have been altered. Secondary quartz is common, filling fractures and coating phenocrysts and xenolithic sediment particles. Plagioclase phenocrysts are completely altered to sericite and calcite. Groundmass plagioclase is not so altered, and albite twinning is visible. Microprobe analysis of the groundmass plagioclase (Maria, ms) indicates values of about An10 and An30 for two of the younger basalts and nearly pure albite for the older basalts. Replacement by highly sodic plagioclase is probably responsible for the lower An values. Pyroxene remains unaltered even when completely surrounded by chlorite, suggesting that interstitial chlorite may represent replaced groundmass (possibly finely crystalline or glassy) rather than altered pyroxene. Because the rocks do not appear to have been metamorphosed, it is probable that much of this alteration is deuteric, though localized alteration resulting from interaction between lava flows and water is also possible.

The two rhyolite flows are mineralogically similar to one another. The phenocryst assemblage consists primarily of feldspar with trace amounts of riebeckite and sphene. Quartz phenocrysts are conspicuously absent. Opaque minerals are very fine-grained and disseminated throughout the groundmass, which is composed of an intergrowth of tiny K-rich feldspar microlaths and quartz. Most of the feldspar phenocrysts range from 0.1 to $0.5 \mathrm{~mm}$ in length. A second population of larger feldspar phenocrysts (2-4 $\mathrm{mm}$ ) forms the subhorizontal strings prominent in hand samples. Both populations consist of anorthoclase, as indicated by euhedral morphology, small extinction angles, and polysynthetic twinning in two directions. Microprobe analyses (Maria, ms) indicate that the phenocrysts have rim compositions of anorthoclase but cores of nearly pure albite. Because there is no evidence of zoning, secondary albitization is suspected. Although there are no quartz phenocrysts, quartz is abundant, filling vesicles and forming the prominent seams that characterize the rhyolite in the field. The sphene and riebeckite crystals are found always in association with the quartz fillings, suggesting that these minerals may represent an alkali-rich liquid or vapor phase released from the lavas during cooling. Small, radially fibrous spherulites (characteristic of devitrification) in flow-banded basal sections and more widespread perlitic textures (fig. 3B) indicate that the rhyolite was partially glassy prior to devitrification.

The pyroclastic units beneath the capping rhyolite consist of lithic and crystal clasts in a matrix composed primarily of devitrified glass shards (fig. 3C) and marked by fine-scale layering. The devitrified shards show curvilinear and wish-bone shapes resembling disrupted vesicle walls. Lithic clasts include fragments (up to $1 \mathrm{~cm}$ ) of rhyolite, basalt, siltstone, or sandstone and exhibit angular or subrounded morphologies. Crystal clasts are angular, broken fragments (up to $2 \mathrm{~mm}$ ) of primarily altered red feldspar and clear quartz. The condition of devitrified shards, molded around and partly enclosing other clasts, suggests that the material was still hot at the time of deposition and may represent pyroclastic flows. One pyroclastic horizon contains feldspar crystals and layers of coalescing spherules (replaced by chalcedony) in a fine-grained, devitrified matrix of brownish crystallites intergrown with dusty opaque minerals (fig. 3D). Tentatively, this unit is interpreted to be an accretionary lapilli tuff. Alternatively, it could be a highly vesiculated variety of obsidian or rhyolite in which the vesicles were subsequently filled with chalcedony.

\section{GEOCHEMISTRY}

Thirteen samples of rhyolite, twenty samples of basalt, and four samples of the pyroclastic rocks were analyzed by X-ray fluorescence for major- and trace-element whole-rock composition (table 1). In addition, two samples of rhyolite and two samples of basalt were analyzed for rare earth elements by neutron activation analysis (table 2). Geochemical analyses support field and petrographic evidence that the volcanic suite 
TABLE 1

Major and trace element data for volcanic units of the Wamsutta Formation

\begin{tabular}{|c|c|c|c|c|c|c|c|c|c|c|c|c|c|}
\hline \multicolumn{14}{|c|}{ Rhyolite } \\
\hline & R-3 & R-8 & R-19A & R-19B & R-26 & R-27 & R-28 & R-29 & R-36 & R-37 & R-39 & R-44 & R-51 \\
\hline \multicolumn{14}{|c|}{ Major Oxides (wt \%) } \\
\hline $\mathrm{SiO}_{2}$ & 73.29 & 73.82 & 70.51 & 76.80 & 73.90 & 72.76 & 74.21 & 73.20 & 72.94 & 73.05 & 75.88 & 73.20 & 75.85 \\
\hline $\mathrm{TiO}_{2}$ & 0.54 & 0.50 & 0.61 & 0.50 & 0.47 & 0.52 & 0.37 & 0.55 & 0.46 & 0.52 & 0.49 & 0.54 & 0.53 \\
\hline $\mathrm{Al}_{2} \mathrm{O}_{3}$ & 13.28 & 12.98 & 15.11 & 11.73 & 13.54 & 13.54 & 12.84 & 13.31 & 13.60 & 13.42 & 12.87 & 13.40 & 13.10 \\
\hline $\mathrm{Fe}_{2} \mathrm{O}_{3}(\mathrm{t})$ & 2.59 & 2.35 & 3.17 & 2.06 & 2.61 & 2.53 & 2.37 & 2.38 & 2.55 & 2.54 & 2.54 & 2.60 & 2.59 \\
\hline $\mathrm{MnO}$ & 0.12 & 0.12 & 0.15 & 0.09 & 0.13 & 0.12 & 0.16 & 0.13 & 0.11 & 0.11 & 0.18 & 0.12 & 0.11 \\
\hline $\mathrm{MgO}$ & 0.21 & 0.06 & 0.10 & 0.57 & 0.28 & 0.21 & 0.33 & 0.19 & 0.02 & 0.20 & 0.44 & 0.29 & 0.10 \\
\hline $\mathrm{CaO}$ & 0.54 & 0.66 & 0.84 & 0.99 & 0.75 & 0.81 & 1.47 & 0.63 & 0.48 & 0.45 & 3.13 & 0.73 & 1.51 \\
\hline $\mathrm{Na}_{2} \mathrm{O}$ & 4.91 & 4.14 & 7.25 & 3.89 & 4.88 & 4.14 & 4.17 & 4.20 & 3.88 & 3.51 & 4.30 & 4.60 & 6.27 \\
\hline $\mathrm{K}_{2} \mathrm{O}$ & 3.66 & 4.23 & 1.06 & 1.90 & 4.43 & 4.10 & 3.78 & 4.20 & 5.06 & 5.50 & 0.60 & 3.76 & 0.55 \\
\hline $\mathrm{P}_{2} \mathrm{O}_{5}$ & 0.06 & 0.05 & 0.07 & 0.07 & 0.06 & 0.05 & 0.05 & 0.07 & 0.09 & 0.05 & 0.07 & 0.07 & 0.05 \\
\hline Total & 99.20 & 98.91 & 98.87 & 98.60 & 101.05 & 98.78 & 99.75 & 98.86 & 99.19 & 99.35 & 100.50 & 99.31 & 100.66 \\
\hline $\mathrm{FeO}(\mathrm{c})$ & 0.49 & 0.31 & 0.95 & 0.05 & 0.58 & 0.46 & 0.45 & 0.30 & 0.53 & 0.47 & 0.49 & 0.50 & 0.50 \\
\hline $\mathrm{FeO}(\mathrm{m})$ & 0.19 & ND & ND & ND & 0.14 & 0.19 & ND & ND & 0.91 & ND & 0.36 & 0.21 & ND \\
\hline \multicolumn{14}{|c|}{ Trace Elements (ppm) } \\
\hline $\mathrm{Rb}$ & 86 & 109 & 34 & 82 & 98 & 82 & 71 & 92 & 121 & 136 & 35 & 98 & 16 \\
\hline $\mathrm{Sr}$ & 46 & 72 & 301 & 282 & 65 & 104 & 116 & 91 & 69 & 52 & 2607 & 104 & 221 \\
\hline $\mathrm{Y}$ & 79 & 74 & 90 & 75 & 81 & 79 & 71 & 79 & 79 & 79 & 69 & 81 & 77 \\
\hline $\mathrm{Zr}$ & 610 & 579 & 688 & 572 & 616 & 623 & 544 & 629 & 607 & 613 & 642 & 633 & 602 \\
\hline $\mathrm{Nb}$ & 52 & 50 & 58 & 49 & 54 & 53 & 46 & 54 & 52 & 54 & 51 & 55 & 51 \\
\hline $\mathrm{Ba}$ & 390 & 577 & 316 & 463 & 531 & 606 & 673 & 588 & 614 & 534 & 182 & 467 & 139 \\
\hline $\mathrm{La}$ & 83 & 95 & 113 & 85 & 99 & 93 & 79 & 94 & 88 & 86 & 87 & 94 & 82 \\
\hline $\mathrm{Ce}$ & 161 & 157 & 185 & 150 & 163 & 161 & 150 & 164 & 163 & 164 & 158 & 177 & 155 \\
\hline $\mathrm{Zn}$ & 135 & 115 & 129 & 122 & 132 & 125 & 144 & 110 & 124 & 137 & 131 & 143 & 108 \\
\hline \multicolumn{14}{|c|}{ CIPW Norms } \\
\hline $\mathrm{Q}$ & 28.75 & 31.57 & 21.67 & 43.65 & 26.26 & 30.41 & 31.63 & 30.67 & 29.49 & 29.80 & 40.89 & 29.58 & 33.62 \\
\hline Or & 21.63 & 25.00 & 6.26 & 11.23 & 26.18 & 24.23 & 22.34 & 24.82 & 29.90 & 32.50 & 3.55 & 22.22 & 3.25 \\
\hline $\mathrm{Ab}$ & 41.55 & 35.03 & 61.35 & 32.92 & 41.29 & 35.03 & 35.29 & 35.54 & 32.83 & 29.70 & 36.39 & 38.92 & 53.06 \\
\hline An & 2.29 & 2.95 & 3.71 & 2.45 & 1.96 & 3.69 & 5.15 & 2.67 & 1.79 & 1.91 & 14.05 & 3.16 & 5.98 \\
\hline $\mathrm{C}$ & 0.40 & 0.51 & 0.68 & 1.64 & 0.00 & 0.94 & 0.00 & 0.88 & 1.08 & 0.99 & 0.00 & 0.60 & 0.00 \\
\hline $\mathrm{Di}$ & 0.00 & 0.00 & 0.00 & 0.00 & 1.17 & 0.00 & 1.53 & 0.00 & 0.00 & 0.00 & 0.86 & 0.00 & 1.03 \\
\hline Hy & 2.00 & 1.49 & 2.14 & 2.47 & 1.73 & 1.98 & 1.69 & 1.78 & 1.60 & 1.95 & 2.29 & 2.20 & 1.18 \\
\hline Mt & 1.25 & 1.13 & 1.53 & 0.99 & 1.26 & 1.22 & 1.14 & 1.15 & 1.23 & 1.23 & 1.23 & 1.26 & 1.25 \\
\hline Il & 1.03 & 0.95 & 1.16 & 0.95 & 0.89 & 0.99 & 0.70 & 1.04 & 0.87 & 0.99 & 0.93 & 1.03 & 1.01 \\
\hline Ap & 0.14 & 0.12 & 0.16 & 0.16 & 0.14 & 0.12 & 0.12 & 0.16 & 0.21 & 0.12 & 0.16 & 0.16 & 0.12 \\
\hline
\end{tabular}

$\mathrm{ND}=$ not determined

$\mathrm{FeO}(\mathrm{m})=$ conc. of $\mathrm{FeO}$ as determined by titration with ferrous ammonium sulfate (Pierce, 1976)

$\mathrm{FeO}(\mathrm{c})=$ calculated value for the concentration of $\mathrm{FeO}$ (Irvine and Baragar, 1971).

$\mathrm{Mg} \#=100\left(\left(\mathrm{Mg}^{+2}\right) /\left(\mathrm{Mg}^{+2}\right)+\left(\mathrm{Fe}^{+2}\right)\right)$, using $\mathrm{FeO}(\mathrm{c})$.

Major and trace elements were determined using a Kevex 0700/7000 energy-dispersive XRF spectrometer.

Samples were analyzed according to Hamidzada (1988). CIPW norms calculated on a wt \% basis using the IGPET II program (Carr, 1987). FeO/Fe2O3 ratios were adjusted according to Irvine and Baragar (1971). 
TABLE 1

(continued)

\begin{tabular}{|c|c|c|c|c|c|c|c|c|c|c|c|c|}
\hline & & & & asalt w & ith no & pyroxe & & & & & Pyrox. & bas. \\
\hline & B-7B & B-7C & B-10 & B-31 & B-32 & B-34 & B-35 & B-41 & B-54 & B-55 & PB-1 & PB-7A \\
\hline Major $\mathrm{O}$ & xides (wt & t \%) & & & & & & & & & & \\
\hline $\mathrm{SiO}_{2}$ & 44.68 & 44.83 & 45.82 & 47.54 & 47.45 & 49.31 & 46.97 & 47.13 & 46.60 & 46.61 & 50.38 & 48.80 \\
\hline $\mathrm{TiO}_{2}$ & 4.55 & 5.22 & 4.31 & 4.25 & 4.24 & 4.00 & 4.09 & 4.20 & 4.30 & 3.99 & 4.47 & 4.84 \\
\hline $\mathrm{Al}_{2} \mathrm{O}_{3}$ & 14.61 & 14.63 & 15.85 & 16.48 & 15.85 & 16.66 & 16.32 & 15.93 & 17.20 & 15.68 & 13.47 & 14.06 \\
\hline $\mathrm{Fe}_{2} \mathrm{O}_{3}(\mathrm{t})$ & 15.62 & 17.23 & 17.92 & 14.36 & 15.83 & 11.32 & 15.32 & 16.50 & 16.14 & 17.82 & 14.85 & 15.01 \\
\hline $\mathrm{MnO}$ & 0.15 & 0.30 & 0.32 & 0.30 & 0.26 & 0.27 & 0.25 & 0.23 & 0.19 & 0.20 & 0.25 & 0.22 \\
\hline $\mathrm{MgO}$ & 6.33 & 6.44 & 6.98 & 6.77 & 5.81 & 5.83 & 6.44 & 6.20 & 5.10 & 4.85 & 4.71 & 4.51 \\
\hline $\mathrm{CaO}$ & 10.09 & 6.68 & 4.37 & 5.50 & 4.01 & 5.71 & 4.51 & 4.56 & 5.56 & 4.81 & 7.56 & 6.92 \\
\hline $\mathrm{Na}_{2} \mathrm{O}$ & 0.61 & 2.81 & 3.91 & 3.44 & 5.35 & 3.98 & 4.17 & 4.33 & 3.19 & 3.85 & 3.50 & 4.13 \\
\hline $\mathrm{K}_{2} \mathrm{O}$ & 2.45 & 1.17 & 0.56 & 1.50 & 0.27 & 0.57 & 0.45 & 0.26 & 1.21 & 1.11 & 0.18 & 0.46 \\
\hline $\mathrm{P}_{2} \mathrm{O}_{5}$ & 1.40 & 1.42 & 0.94 & 0.91 & 0.88 & 0.82 & 0.87 & 0.89 & 0.93 & 0.89 & 1.27 & 1.37 \\
\hline Total & 100.49 & 100.73 & 100.98 & 101.05 & 99.95 & 98.47 & 99.39 & 100.23 & 100.42 & 99.81 & 100.64 & 100.32 \\
\hline $\mathrm{FeO}(\mathrm{c})$ & 8.61 & 9.46 & 10.90 & 7.75 & 9.08 & 5.24 & 8.76 & 9.72 & 9.30 & 11.10 & 7.99 & 7.80 \\
\hline $\mathrm{FeO}(\mathrm{r}$ & ND & ND & 10.15 & 5.43 & 6.76 & ND & 7.26 & ND & ND & ND & 8.25 & ND \\
\hline $\mathrm{Mg} \#$ & 56.72 & 54.82 & 53.30 & 60.89 & 53.28 & 66.48 & 56.72 & 53.20 & 49.43 & 43.78 & 51.24 & 50.75 \\
\hline Trace & ents ( & (ppm) & & & & & & & & & & \\
\hline $\mathrm{Rb}$ & 67 & 32 & 18 & 52 & 7 & 25 & 13 & 8 & 30 & 34 & 6 & 17 \\
\hline $\mathrm{Sr}$ & 83 & 309 & 366 & 448 & 598 & 510 & 556 & 456 & 326 & 657 & 564 & 655 \\
\hline Y & 35 & 41 & 35 & 37 & 35 & 34 & 37 & 34 & 38 & 68 & 36 & 42 \\
\hline $\mathrm{Zr}$ & 231 & 260 & 292 & 292 & 297 & 270 & 288 & 292 & 306 & 312 & 230 & 258 \\
\hline $\mathrm{Nb}$ & 30 & 34 & 34 & 33 & 33 & 30 & 33 & 33 & 36 & 35 & 29 & 32 \\
\hline $\mathrm{Ba}$ & 456 & 285 & 244 & 473 & 312 & 230 & 191 & 248 & 443 & 540 & 126 & 260 \\
\hline $\mathrm{La}$ & 32 & 45 & 39 & 26 & 41 & 40 & 38 & 34 & 34 & 35 & 44 & 41 \\
\hline $\mathrm{Ce}$ & 75 & 84 & 76 & 66 & 76 & 71 & 76 & 71 & 75 & 75 & 73 & 81 \\
\hline $\mathrm{Zn}$ & 196 & 191 & 169 & 161 & 147 & 141 & 151 & 152 & 188 & 152 & 157 & 168 \\
\hline & & & & & & & & & & & & \\
\hline Q & 5.40 & 2.39 & 0.00 & 1.11 & 0.00 & 5.37 & 1.83 & 1.43 & 3.99 & 0.56 & 10.29 & 6.14 \\
\hline Or & 14.48 & 6.91 & 3.31 & 8.86 & 1.60 & 3.37 & 2.66 & 1.54 & 7.15 & 6.56 & 1.06 & 2.72 \\
\hline $\mathrm{Ab}$ & 5.16 & 23.78 & 33.09 & 29.11 & 45.27 & 33.68 & 35.29 & 36.64 & 26.99 & 32.58 & 29.62 & 34.95 \\
\hline An & 29.89 & 23.85 & 15.54 & 21.34 & 14.14 & 22.97 & 16.69 & 16.81 & 21.51 & 18.05 & 20.51 & 18.47 \\
\hline $\mathrm{C}$ & 0.00 & 0.00 & 3.12 & 1.38 & 1.57 & 1.08 & 2.86 & 2.37 & 2.76 & 1.53 & 0.00 & 0.00 \\
\hline $\mathrm{Di}$ & 8.76 & 0.01 & 0.00 & 0.00 & 0.00 & 0.00 & 0.00 & 0.00 & 0.00 & 0.00 & 6.92 & 5.45 \\
\hline Hy & 15.19 & 19.79 & 25.56 & 19.88 & 12.95 & 14.52 & 21.21 & 22.07 & 18.25 & 21.70 & 11.27 & 10.17 \\
\hline $\mathrm{Ol}$ & 0.00 & 0.00 & 0.37 & 0.00 & 4.99 & 0.00 & 0.00 & 0.00 & 0.00 & 0.00 & 0.00 & 0.00 \\
\hline Mt & 8.77 & 9.74 & 8.42 & 8.34 & 8.32 & 6.17 & 8.10 & 8.26 & 8.41 & 7.96 & 8.66 & 9.19 \\
\hline Il & 8.64 & 9.91 & 8.19 & 8.07 & 8.05 & 7.60 & 7.77 & 7.98 & 8.17 & 7.58 & 8.49 & 9.19 \\
\hline Hem & 0.00 & 0.00 & 0.00 & 0.00 & 0.00 & 1.24 & 0.00 & 0.00 & 0.00 & 0.00 & 0.00 & 0.00 \\
\hline Ap & 3.24 & 3.29 & 2.18 & 2.11 & 2.04 & 1.90 & 2.02 & 2.06 & 2.15 & 2.06 & 2.94 & 3.17 \\
\hline
\end{tabular}


TABLe 1

(continued)

\begin{tabular}{|c|c|c|c|c|c|c|c|c|}
\hline \multicolumn{9}{|c|}{ Pyroxene basalt } \\
\hline & PB-11 & PB-12 & PB-17 & PB-30 & PB-38 & PB-46 & PB-47 & PB-50 \\
\hline \multicolumn{9}{|c|}{ Major Oxides (wt \%) } \\
\hline $\mathrm{SiO}_{2}$ & 49.54 & 47.57 & 46.34 & 48.21 & 46.29 & 51.68 & 47.81 & 46.57 \\
\hline $\mathrm{TiO}_{2}$ & 4.81 & 4.84 & 5.21 & 3.69 & 2.80 & 2.80 & 2.72 & 3.14 \\
\hline $\mathrm{Al}_{2} \mathrm{O}_{3}$ & 13.91 & 13.87 & 14.73 & 15.58 & 15.52 & 14.39 & 14.73 & 16.39 \\
\hline $\mathrm{Fe}_{2} \mathrm{O}_{3}(\mathrm{t})$ & 13.51 & 16.33 & 14.78 & 15.18 & 14.40 & 14.69 & 14.83 & 16.72 \\
\hline $\mathrm{MnO}$ & 0.24 & 0.21 & 0.28 & 0.19 & 0.23 & 0.23 & 0.18 & 0.21 \\
\hline $\mathrm{MgO}$ & 4.93 & 4.69 & 6.10 & 5.85 & 6.54 & 5.72 & 5.52 & 4.43 \\
\hline $\mathrm{CaO}$ & 7.16 & 7.33 & 6.85 & 6.08 & 9.29 & 5.89 & 9.55 & 6.13 \\
\hline $\mathrm{Na}_{2} \mathrm{O}$ & 4.27 & 4.31 & 4.75 & 4.28 & 3.12 & 3.11 & 3.09 & 3.59 \\
\hline $\mathrm{K}_{2} \mathrm{O}$ & 0.23 & 0.21 & 0.22 & 0.28 & 0.97 & 0.61 & 0.89 & 2.30 \\
\hline $\mathrm{P}_{2} \mathrm{O}_{5}$ & 1.41 & 1.44 & 1.48 & 0.74 & 0.60 & 0.59 & 0.59 & 0.67 \\
\hline Total & 100.01 & 100.80 & 100.74 & 100.08 & 99.76 & 99.71 & 99.91 & 100.15 \\
\hline $\mathrm{FeO}(\mathrm{c})$ & 6.48 & 8.99 & 7.26 & 8.99 & 9.09 & 9.35 & 9.57 & 10.87 \\
\hline $\mathrm{FeO}(\mathrm{m})$ & ND & 8.54 & 6.58 & ND & ND & 3.11 & 8.27 & ND \\
\hline $\mathrm{Mg} \#$ & 57.56 & 48.18 & 59.96 & 53.70 & 56.19 & 52.16 & 50.69 & 42.08 \\
\hline \multicolumn{9}{|c|}{ Trace Elements (ppm) } \\
\hline $\mathrm{Rb}$ & 6 & 4 & 4 & 9 & 21 & 18 & 24 & 59 \\
\hline $\mathrm{Sr}$ & 507 & 662 & 358 & 713 & 912 & 680 & 989 & 567 \\
\hline $\mathrm{Y}$ & 41 & 37 & 43 & 30 & 26 & 25 & 26 & 32 \\
\hline $\mathrm{Zr}$ & 255 & 258 & 265 & 253 & 194 & 190 & 198 & 221 \\
\hline $\mathrm{Nb}$ & 31 & 31 & 34 & 28 & 16 & 17 & 17 & 21 \\
\hline $\mathrm{Ba}$ & 209 & 272 & 162 & 500 & 477 & 491 & 575 & 611 \\
\hline $\mathrm{La}$ & 38 & 41 & 47 & 34 & 32 & 25 & 28 & 33 \\
\hline $\mathrm{Ce}$ & 79 & 80 & 88 & 69 & 64 & 59 & 61 & 71 \\
\hline $\mathrm{Zn}$ & 165 & 156 & 178 & 138 & 127 & 136 & 112 & 115 \\
\hline \multicolumn{9}{|c|}{ CIPW Norms } \\
\hline Q & 6.88 & 3.35 & 0.00 & 0.25 & 0.00 & 7.88 & 0.00 & 0.00 \\
\hline Or & 1.36 & 1.24 & 1.30 & 1.65 & 5.73 & 3.60 & 5.26 & 13.59 \\
\hline $\mathrm{Ab}$ & 36.13 & 36.47 & 40.19 & 36.22 & 26.40 & 26.32 & 26.15 & 30.38 \\
\hline An & 18.11 & 17.88 & 18.22 & 22.47 & 25.48 & 23.50 & 23.70 & 21.28 \\
\hline $\mathrm{C}$ & 0.00 & 0.00 & 0.00 & 0.00 & 0.00 & 0.00 & 0.00 & 0.00 \\
\hline $\mathrm{Di}$ & 6.38 & 7.26 & 4.74 & 2.30 & 13.55 & 1.52 & 16.23 & 3.49 \\
\hline Hy & 9.32 & 11.88 & 11.62 & 19.94 & 4.04 & 22.93 & 12.64 & 0.80 \\
\hline $\mathrm{Ol}$ & 0.00 & 0.00 & 0.96 & 0.00 & 10.61 & 0.00 & 2.23 & 14.62 \\
\hline Mt & 7.73 & 9.19 & 9.22 & 7.53 & 6.23 & 6.23 & 6.12 & 6.73 \\
\hline Il & 9.14 & 9.19 & 9.90 & 7.01 & 5.32 & 5.32 & 5.17 & 5.96 \\
\hline Hem & 0.98 & 0.00 & 0.35 & 0.00 & 0.00 & 0.00 & 0.00 & 0.00 \\
\hline Ap & 3.27 & 3.34 & 3.43 & 1.71 & 1.39 & 1.37 & 1.37 & 1.55 \\
\hline
\end{tabular}

Pyroclastic rocks

PY-5 PY-9 PY-10 PY-11

$\begin{array}{llll}65.56 & 73.06 & 73.76 & 76.16\end{array}$

$\begin{array}{llll}0.86 & 0.72 & 0.57 & 0.45\end{array}$

$\begin{array}{llll}17.38 & 13.38 & 13.4 & 12.01\end{array}$

$\begin{array}{llll}3.97 & 4.15 & 2.99 & 1.68\end{array}$

$\begin{array}{llll}0.1 & 0.12 & 0.1 & 0.11\end{array}$

$\begin{array}{llll}2.56 & 2.39 & 2.58 & 2.23\end{array}$

$\begin{array}{llll}1.08 & 0.87 & 0.57 & 1.7\end{array}$

$\begin{array}{llll}1.92 & 2.68 & 2.94 & 1.16\end{array}$

$\begin{array}{llll}4.94 & 3.08 & 2.2 & 2.81\end{array}$

$\begin{array}{llll}0.1 & 0.09 & 0.13 & 0.07\end{array}$

$\begin{array}{lllll}98.47 & 100.54 & 99.24 & 98.38\end{array}$

\begin{tabular}{rrrr}
179 & 123 & 76 & 112 \\
171 & 125 & 147 & 97 \\
81 & 77 & 81 & 65 \\
804 & 581 & 542 & 479 \\
71 & 51 & 62 & 57 \\
650 & 432 & 357 & 351 \\
138 & 64 & 70 & 76 \\
211 & 116 & 103 & 74 \\
180 & 184 & 191 & 165 \\
\hline
\end{tabular}


TABLE 2

Rare earth and trace elements determined by neutron activation analysis

\begin{tabular}{|c|c|c|c|c|}
\hline & R-36 & ${ }^{*} \mathbf{R}-39$ & B-31 & PB-47 \\
\hline \multicolumn{5}{|c|}{ Rare Earth Elements (ppm) } \\
\hline $\mathrm{La}$ & 77.71 & 71.18 & 30.35 & 26.74 \\
\hline $\mathrm{Ce}$ & 161.25 & 152.81 & 70.47 & 58.57 \\
\hline $\mathrm{Nd}$ & 78.31 & 67.69 & 29.7 & 19.11 \\
\hline $\mathrm{Sm}$ & 15.82 & 14.13 & 8.9 & 6.82 \\
\hline $\mathrm{Eu}$ & 3.15 & 2.91 & 2.84 & 2.2 \\
\hline $\mathrm{Tb}$ & 2.69 & 2.11 & 1.38 & 1.06 \\
\hline $\mathrm{Yb}$ & 6.99 & 6.48 & 2.77 & 1.98 \\
\hline $\mathrm{Lu}$ & 0.898 & 0.858 & 0.468 & 0.459 \\
\hline \multicolumn{5}{|c|}{ Trace Elements (ppm) } \\
\hline $\mathrm{Hf}$ & 14.26 & 13.09 & 6.53 & 4.29 \\
\hline $\mathrm{Ta}$ & 3.37 & 3.06 & 2.18 & 1.25 \\
\hline $\mathrm{Sb}$ & 0.31 & 0.17 & ND & ND \\
\hline $\mathrm{U}$ & 2.26 & 3.87 & ND & ND \\
\hline Th & 11.72 & 10.69 & 2.39 & 1.74 \\
\hline Co & 0.42 & 0.48 & 48.16 & 42.09 \\
\hline $\mathrm{Cr}$ & n.d. & 2.4 & 13.52 & 46.5 \\
\hline $\mathrm{Sc}$ & 7.47 & 7.06 & 19.43 & 29.51 \\
\hline Cs & 0.98 & n.d. & 1.62 & 0.88 \\
\hline
\end{tabular}

* R-39 is basal vitrophyre.

is bimodal (table 1; fig. 4). On a plot of total alkalies versus silica (fig. 4), the Wamsutta volcanics fall primarily within the fields of basalt and rhyolite. Rocks containing between 52 to $70 \mathrm{wt}$ percent silica are absent. In addition, it is apparent that these rocks are transitionally or moderately alkaline (fig. 4). The moderately alkaline nature of the volcanics is also reflected by their mineralogic and trace element compositions.

The older, pyroxene-free basalts and the younger, pyroxene-bearing basalts have very similar major-element compositions (table 1 ). The older basalts typically are slightly richer in $\mathrm{Al}, \mathrm{Mg}, \mathrm{Fe}, \mathrm{P}$, and $\mathrm{Ti}$ and poorer in $\mathrm{Ca}$ and $\mathrm{Si}$ (fig. 5). Relative to tholeiites, both basalts are low in $\mathrm{Mg}$ and Ca while high in $\mathrm{Ti}, \mathrm{Fe}, \mathrm{Mn}, \mathrm{Na}, \mathrm{K}$, and $\mathrm{P}$. $\mathrm{SiO}_{2}$ levels range from 44 to 52 percent. Low $\mathrm{K}_{2} \mathrm{O} / \mathrm{Na}_{2} \mathrm{O}$ ratios, low $\mathrm{MgO}$, and high $\mathrm{P}_{2} \mathrm{O}_{5}$ and $\mathrm{TiO}_{2}$ levels suggest that the Wamsutta basalts are hawaiites. This classification is consistent with the Na-rich groundmass plagioclase and the high concentrations of $\mathrm{TiO}_{2}$ (1.77-2.61 percent), $\mathrm{Na}_{2} \mathrm{O}$ (.39-.50 percent), and $\mathrm{Al}_{2} \mathrm{O}_{3}$ (3.1-4.1 percent) in the clinopyroxene (Maria, ms). High $\mathrm{Al}$ reflects the undersaturated nature of most alkaline basalts, as $\mathrm{Al}$ is incorporated into the pyroxene if there is insufficient $\mathrm{Si}$ (Kushiro, 1960; Le Bas, 1962). However, the altered state of the Wamsutta basalts makes classification using major elements questionable. Inconsistent normative values for quartz, olivine, and hypersthene (table 1) illustrate the effects of secondary processes, and low diopside values, even for samples with abundant pyroxene, suggest loss of alkalies.

Less-mobile trace elements (high-field-strength elements) are more suitable for characterizing the Wamsutta volcanics. The pyroxene-bearing and pyroxene-free 


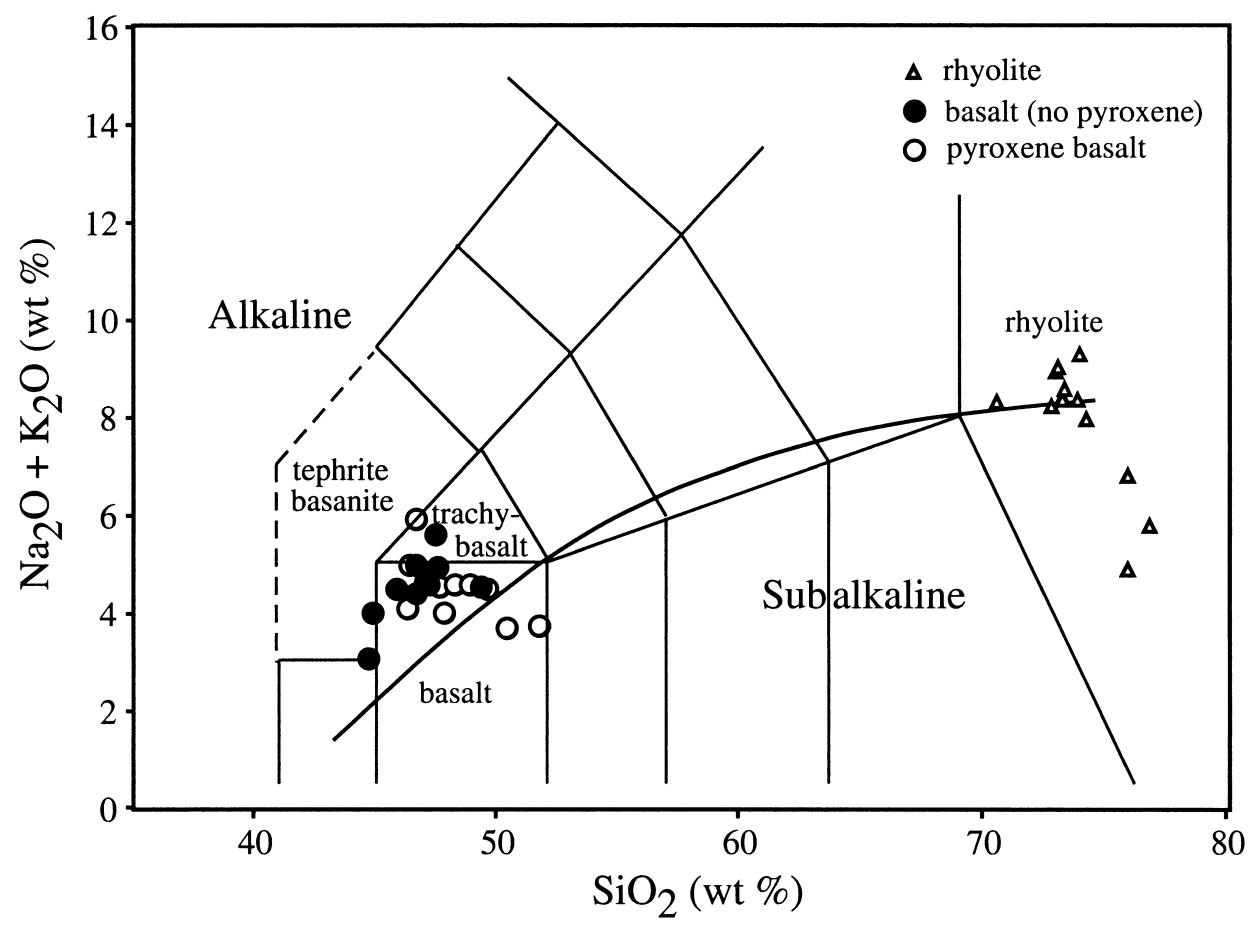

Fig. 4. Plot of $\mathrm{K}_{2} \mathrm{O}+\mathrm{Na}_{2} \mathrm{O}$ versus $\mathrm{SiO}_{2}$ for the Wamsutta volcanics. Fields and rock names are after LeBas and others, 1986. The dividing line between alkaline and subalkaline rocks is from Miyashiro, 1978. The rocks of this bimodal suite fall primarily within the fields of basalt and rhyolite. It is also apparent that the volcanics are moderately alkaline.

basalts are both enriched in $\mathrm{Zr}, \mathrm{Y}, \mathrm{Nb}$, Ce, and $\mathrm{La}$ (table 1; fig. 5), with levels comparable to those in some alkaline olivine basalts. The ratio of $\mathrm{Nb} / \mathrm{Y}$ is only slightly less than one and is typical of transitional alkaline basalts (Winchester and Floyd, 1977). On average, the older basalts tend to be slightly richer in $\mathrm{Zr}, \mathrm{Ni}, \mathrm{Zn}, \mathrm{Y}$, and $\mathrm{Nb}$. Both basalts show the overall enrichment in REE and enrichment of light relative to heavy REE (table 2; fig. 6) characteristic of alkaline rocks. The REE patterns show no $\mathrm{Eu}$ anomalies (fig. 6). Both basalt types, with the exception of one point, fall in the within-plate alkaline field (fig. 7) of Meschede, 1986. High $\mathrm{Ti} / \mathrm{Y}$ and $\mathrm{Zr} / \mathrm{Y}$ ratios also mark them as within-plate basalts (Pearce and Cann, 1973; Floyd and Winchester, 1975; Pearce and Gale, 1977; Pearce and Norry, 1979). On a plot of trace element variation (fig. 8A), the Wamsutta basalts show relatively high levels of incompatible elements and prominent peaks at $\mathrm{P}$ and Ti. The basalts (particularly the pyroxene-free basalt) follow the within-plate alkalic trend quite closely (with the exception of $\mathrm{Nb}$, $\mathrm{Ta}$, and $\mathrm{Ce}$ ) and are distinct from the within-plate tholeiite. The transitional character of the basalt is demonstrated by its similarity to the Naivasha transitional basalt of the Kenya Rift Valley (Davies and Macdonald, 1987).

The two rhyolite flows are geochemically similar, with relatively high concentrations of Si (71-77 percent), Ti, Fe, and Mn, and low Ca (table 1; fig. 5). Based on levels of $\mathrm{Al}$ and $\mathrm{Fe}$, the Wamsutta rhyolite falls into the comendite category ( $>11$ percent $\mathrm{Al}_{2} \mathrm{O}_{3},<4$ percent $\mathrm{FeO}$ ) of alkaline rhyolites (Macdonald and Bailey, 1973; Gwinn and Hess, 1989). However, while the presence of anorthoclase and riebeckite is an indication of alkalinity (Sutherland, 1974), the Wamsutta rhyolite does not contain 

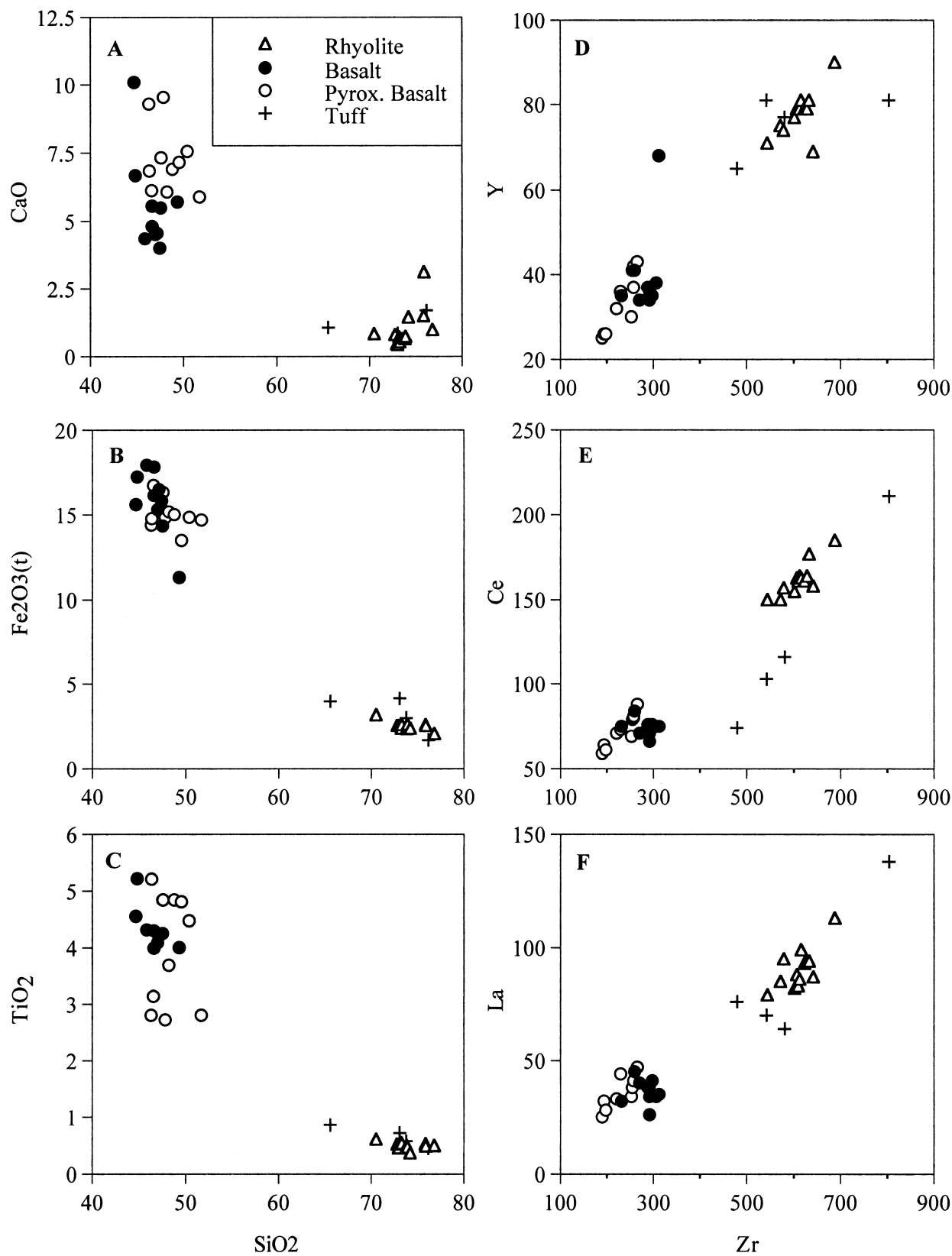

Fig. 5. Variation diagrams plotting major elements versus $\mathrm{SiO}_{2}(\mathrm{~A}, \mathrm{~B}$, and $\mathrm{C}$ ) and trace elements versus $\mathrm{Zr}(\mathrm{D}, \mathrm{E}$, and $\mathrm{F}$ ) for the Wamsutta volcanics. Relative to tholeiites, the basalts are low in $\mathrm{Mg}$ and $\mathrm{Ca}$ while high in $\mathrm{Ti}, \mathrm{Fe}, \mathrm{Mn}, \mathrm{K}$, and $\mathrm{P}$. The basalts are enriched in $\mathrm{Zr}, \mathrm{Y}, \mathrm{Nb}, \mathrm{Ce}$, and $\mathrm{La}$, with levels comparable to those in some alkaline olivine basalts. The rhyolite may be characterized as highly silicic, rich in $\mathrm{Ti}, \mathrm{Fe}$, and $\mathrm{Mn}$, and low in Ca. The rhyolite is enriched in $\mathrm{Zr}, \mathrm{Nb}, \mathrm{Y}, \mathrm{Ce}$, and La.

normative acmite (acmite indicates an excess of $\mathrm{Na}$ over $\mathrm{Al}$ after the formation of albite), unlike most alkaline rhyolites. This absence could be explained by an abundance of $\mathrm{Cl}$ and $\mathrm{F}$ in the magma, which can tie up $\mathrm{Na}$ as $\mathrm{NaCl}$ and $\mathrm{NaF}$ (Macdonald, 


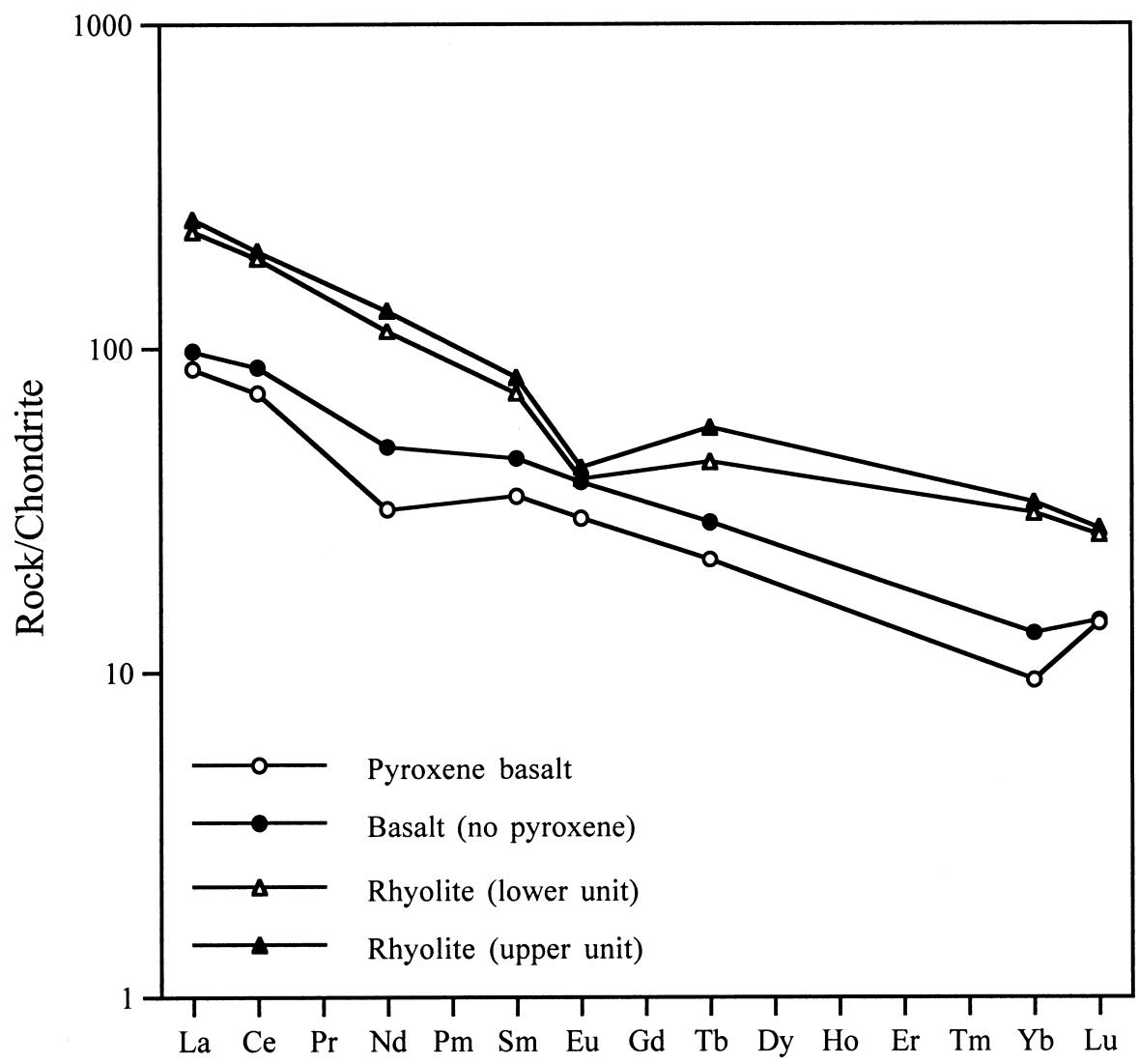

Atomic Number

Fig. 6. REE patterns for the Wamsutta volcanics (normalized to chondrite values from Boynton, 1984). The basalts and rhyolite exhibit overall enrichment in REE and relative enrichment of light to heavy REE. Neither rock type shows a strong Eu anomaly.

1974a). The restriction of riebeckite to quartz-filled seams and vesicles suggests that a late-stage, fluid-rich phase may have scavenged much of the Na. Mobilization of alkali elements is also suggested by normative corundum, which indicates insufficient $\mathrm{K}, \mathrm{Na}$, and $\mathrm{Ca}$ to tie up $\mathrm{Al}$ during the formation of feldspars. Loss of $\mathrm{Na}, \mathrm{Ca}$, and $\mathrm{K}$ is actually characteristic of alkaline rhyolites during their final stages of crystallization and is even more pronounced for glassy rocks (Noble, 1970). Changes in chemistry near the basal contact of the rhyolite (depletion of $\mathrm{K}$ and irregularly high Sr contents-table 1, samples R-19B and R-39) are consistent with exposure to groundwater (Noble, 1970) or extrusion onto wet sediment.

Despite the absence of normative acmite, the concentrations of less-mobile major elements (high $\mathrm{Fe}, \mathrm{Mn}$, and $\mathrm{Ti}$, and low $\mathrm{Al}$ ) distinguish the Wamsutta rhyolite from subalkaline rhyolites (Macdonald, 1974a). High levels of $\mathrm{Fe}^{+3}$ and $\mathrm{Ti}^{+4}$ are typical of alkaline rhyolites because highly charged cations are stabilized by concentrations of alkalies in excess of that required to charge balance $\mathrm{Al}$ (Leat and others, 1986; Gwinn and Hess, 1989). The rhyolite is also enriched in highly charged trace elements such as $\mathrm{Zr}, \mathrm{Nb}, \mathrm{Y}, \mathrm{Ce}$, and La (fig. 5). However, it is considerably less enriched in incompatible 


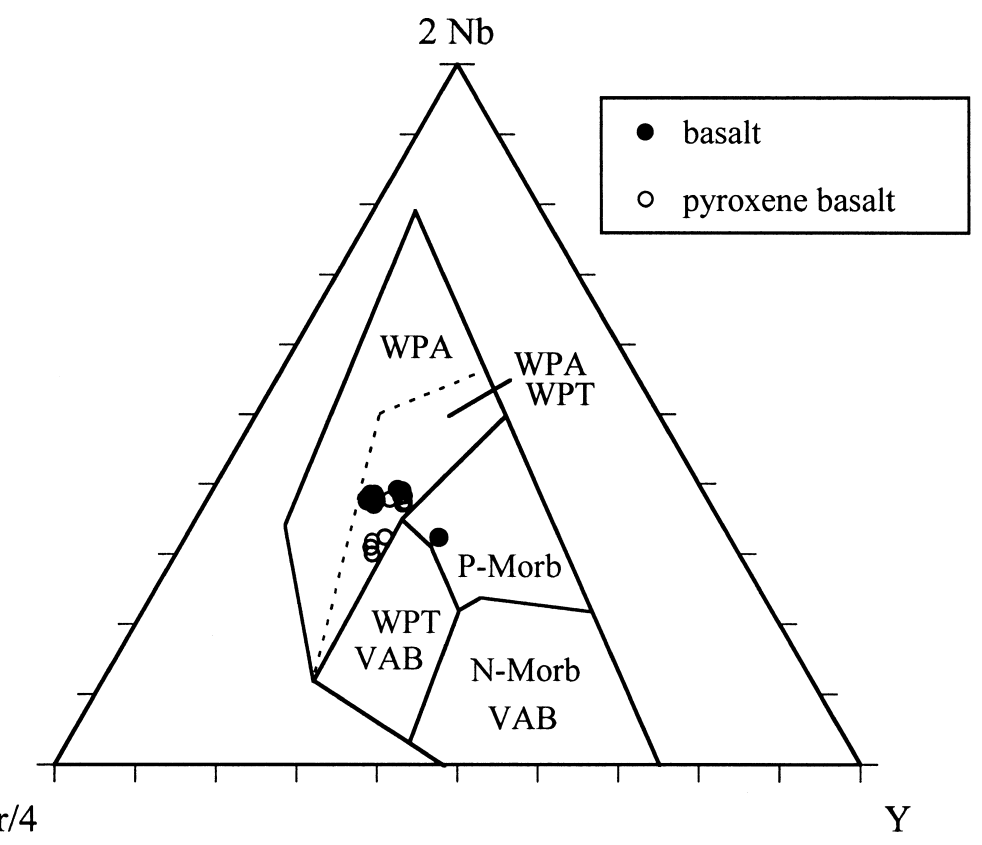

Fig. 7. On a plot of $2 \mathrm{Nb}$ versus $\mathrm{Y}$ versus. $\mathrm{Zr} / 4$ (Meschede, 1986), the basalts fall in the within-plate alkaline field.

and high-field-strength elements ( $\mathrm{Zr}, \mathrm{Y}, \mathrm{Nb}$, and $\mathrm{Zn}$ ) than nearby alkaline volcanics of the Spencer Hill and Blue Hill complexes (Hermes and Murray, 1990; Hermes and Ritchie, 1997). The rhyolite's REE pattern (table 2; fig. 6) shows the enrichment of light relative to heavy REE characteristic of alkaline rocks. Compared to the basalts, the rhyolite shows greater overall enrichment of REE than the basalts and a small Eu anomaly. High concentrations of $\mathrm{Nb}$ and $\mathrm{Y}$ put the Wamsutta rhyolite in the withinplate granite field of Pearce and others (1984; illustrated in Maria, ms), consistent with the within-plate nature of the basalts. On a plot of trace element variation (fig. 8B), the Wamsutta rhyolite shows relative enrichment in REE and $\mathrm{Zr}$ and negative peaks at $\mathrm{Sr}$, $\mathrm{P}$, and $\mathrm{Ti}$. These same characteristics are shared by the pantellerite (fig. $8 \mathrm{~B}$ ), though the peaks are larger. Like the Wamsutta basalts, the rhyolite is geochemically similar to transitional rocks from the Naivasha suite of the Kenya Rift Valley (the transitional comendite, Macdonald and others, 1987).

The major element characteristics of the pyroclastic rocks are generally similar to those of the rhyolite (based on four analyses-table 1; fig. 5), with a tendency to exhibit more intermediate compositions due to included components of basaltic and nonvolcanic lithic clasts. In comparison with the rhyolite, the pyroclastic rocks exhibit a greater range in $\mathrm{SiO}_{2}$ and higher $\mathrm{MgO}$ concentrations. With respect to trace elements, the pyroclastic rocks show a wider range of $\mathrm{Zr}$ concentrations and considerably more scatter in Ce (fig. 5E) and La (fig. 5F) than the rhyolite. It is likely that some of these discrepancies are related to contamination by lithic fragments.

DISCUSSION

Petrogenesis.-The mineralogic and geochemical data indicate that the bimodal Wamsutta volcanic suite is mildly alkaline and was formed in a continental within-plate, rift-related setting. Although the distinctive traits of the Wamsutta basalt and rhyolite 

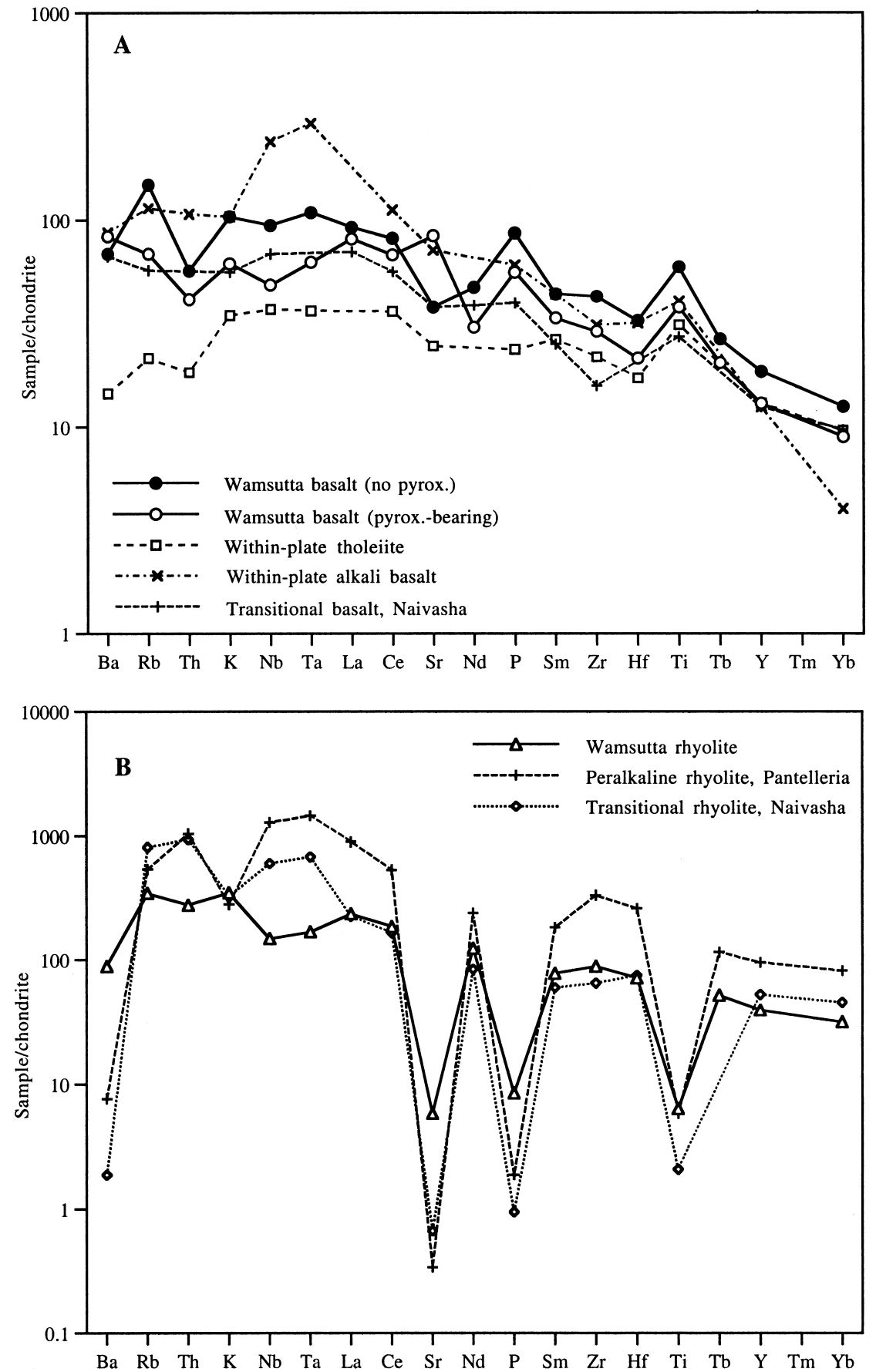

Fig. 8(A) Chondrite-normalized trace element concentrations of basalts plotted from left to right in order of increasing compatibility in a small fraction melt of the mantle. The normalizing values are those of Thompson (1982). Compositions for the within-plate tholeiite, within-plate alkali basalt, and calcalkaline basalt are from Pearce (1982). The transitional basalt composition, Naivasha, is from Davies and Macdonald (1987). (B) Chondrite-normalized trace element concentrations of rhyolites plotted from left to right in order of increasing compatibility in a small fraction melt of the mantle. The normalizing values are those of Thompson (1982). Composition of the calcalkaline rhyolite, Taupo, New Zealand, is from Ewart (1979). The peralkaline rhyolite composition, Pantelleria, is from Civetta and others (1984). The transitional rhyolite composition, Naivasha, is from Macdonald and others (1987). 
probably reflect a complex mixture of petrogenetic factors, some basic interpretations can be made regarding their origins. The bimodality of the suite, with total absence of intermediate compositions, strongly suggests separate sources for the mafic and felsic magmas (fig. 5). Trace element concentrations further discount the possibility that the mafic and felsic rocks are endmembers of a comagmatic series. Basalts and rhyolites form distinct clusters with different trends on a plot of two highly incompatible elements ( $\mathrm{Zr}$ versus $\mathrm{Nb}$, fig 9), suggesting different magma sources. A single trend would be expected if rhyolite and basalt were comagmatic and related by fractional crystallization or magma mixing. ${ }^{13}$

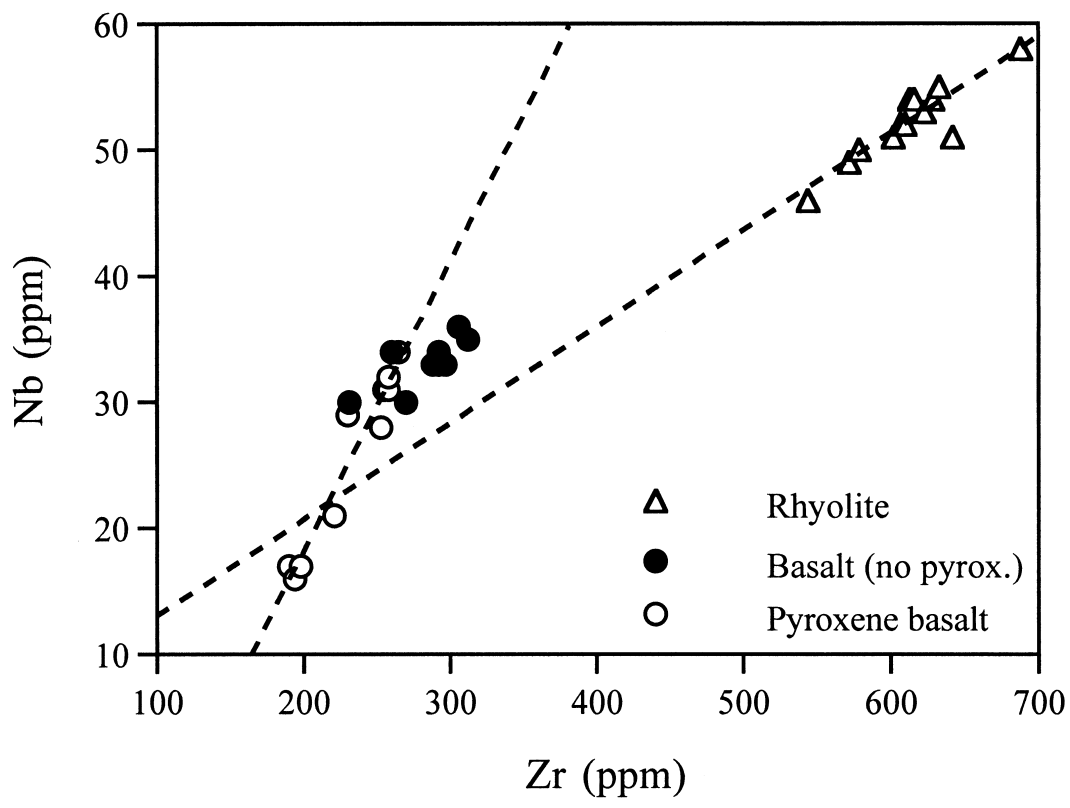

Fig. 9. Plot of $\mathrm{Nb}$ versus $\mathrm{Zr}$ for the Wamsutta volcanics. Limited trace-element trends are consistent with enrichment of incompatible elements by limited fractionation. The different slopes of the trends are consistent with origins for the basalts and rhyolites by partial melting of different sources.

A mantle source similar to that of ocean-island basalts is suggested for the Wamsutta basalts based on their position on plots of $\mathrm{Yb} / \mathrm{Ta}$ versus $\mathrm{Y} / \mathrm{Nb}$, and $\mathrm{Ce} / \mathrm{Nb}$ versus $\mathrm{Y} / \mathrm{Nb}$ (Eby, 1992), within or near the ocean-island basalt field (fig. 10). Low $\mathrm{Mg}$ values $\left[100 \mathrm{Mg} /\left(\mathrm{Mg}+\mathrm{Fe}^{+2}\right)\right]$, ranging from 42 to 67 (table 1), with most at about 53, indicate that the basalts are not primary. Phenocrysts of olivine (now pseudomorphed) and plagioclase in the basalts suggest that fractionation probably played some role in their formation. However, major-element plots (fig. 5) show no compositional trends that might indicate the extent of fractionation and phases involved. High levels of Fe, $\mathrm{Ti}$, and $\mathrm{P}$ suggest that fractionation of $\mathrm{Fe}-\mathrm{Ti}$ oxides and apatite was only minor. Similarly, high levels of $\mathrm{Al}$ and $\mathrm{Sr}$ suggest little fractionation of plagioclase. Some of the trace elements show small trends (for example, $\mathrm{Y}$ versus $\mathrm{Zr}$ in fig. $5 \mathrm{D}$ and $\mathrm{Nb}$ versus $\mathrm{Zr}$ in fig. 9) but nothing that would indicate extensive fractionation. Finally, significant loss of plagioclase would have resulted in an Eu anomaly, which is absent (fig. 6). Slightly higher concentrations of incompatible elements (figs. 6 and 8A) and lower concentrations of $\mathrm{Ba}$ and $\mathrm{Sr}$ (consistent with the removal of plagioclase) in the older 


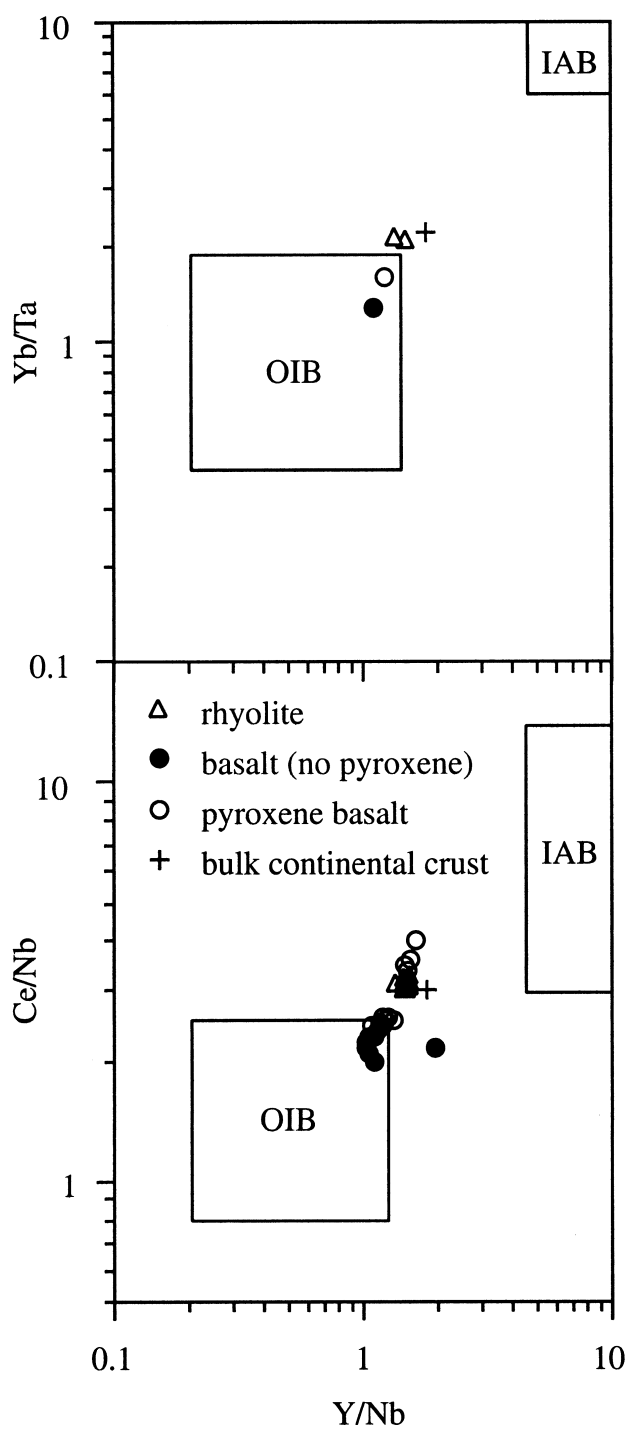

Fig. 10. Plots of $\mathrm{Yb} / \mathrm{Ta}$ versus $\mathrm{Y} / \mathrm{Nb}$ and $\mathrm{Ce} / \mathrm{Nb}$ versus $\mathrm{Y} / \mathrm{Nb}$ after Eby (1992). The basalts fall within or near the ocean-island basalt field, suggesting a mantle source similar to that of ocean-island basalts. The rhyolite falls very near the estimated composition for bulk continental crust (Taylor and McLennan, 1985).

basalts might indicate that they experienced more fractionation than the younger. However, this interpretation is inconsistent with the higher magma temperatures (above the pyroxene liquidus) implied for the older basalts by their lack of pyroxene. The lower $\mathrm{Ba}$ and $\mathrm{Sr}$ concentrations are likely the result of alteration.

It is probable that the basalts ascended rapidly, with little time for fractionation. The absence of pyroxene in the two earliest basalt flows suggests that the magma was maintained at a temperature above the pyroxene liquidus and then quenched. Later basalts, though cool enough to crystallize pyroxene, were also chilled rapidly as demonstrated by the subophitic pyroxene textures and feather-like plagioclase morphologies. The Ti-rich composition of the augite is consistent with crystallization at 
relatively low pressure (Wilkinson, 1974). Clearly, extensive crystal fractionation cannot be called on to explain the enriched levels of incompatible elements in these basalts. An enriched source and small degree of partial melting are more likely explanations. The enrichment of light relative to heavy REE (fig. 6) suggests derivation from a non-depleted source, and heavy REE concentrations 10 to 20 times chondritic values are consistent with a shallow to modest depth of origin from garnet-free mantle (Wilson, 1989). The slightly higher concentrations of incompatible elements in the older basalts might indicate that they represent smaller batches of melt or simply that the source was more depleted when the younger basalts were produced. Alternatively, the slightly lower concentrations of incompatible elements in the younger basalts might reflect interaction with continental crust.

Derivation of rhyolitic magma is usually attributed to fractionation of a more mafic parent or partial melting of crustal rocks. Like the basalts, it appears that formation of the Wamsutta rhyolite involved crystal fractionation only to a limited extent. The presence of anorthoclase as phenocrysts suggests that it may have been a fractionating phase, and linear trends exhibited in variation diagrams (figs. $5 \mathrm{D}$, E, and 9) are consistent with the enrichment of incompatible elements by limited fractionation. However, the small size of the Eu anomaly (fig. 6) as well as relatively high Ba and Sr concentrations (fig. 8B) suggest that feldspar fractionation did not occur to the extent required to derive rhyolite from basalt. Discordant slopes of trace element trends for the Wamsutta rhyolite and basalt (fig. 9) suggest they were derived by partial melting of different sources. The rapid, alternating effusion of basalt and rhyolite and the absence of intermediate rocks are also consistent with separate sources. A crustal source is most likely for the rhyolite. On plots of $\mathrm{Yb} / \mathrm{Ta}$ versus $\mathrm{Y} / \mathrm{Nb}$ and $\mathrm{Ce} / \mathrm{Nb}$ versus $\mathrm{Y} / \mathrm{Nb}$ (fig. 10), the rhyolite falls very near the estimated composition for bulk continental crust (Taylor and McLennan, 1985; Eby, 1992).

Given the full spectrum of incompatible element enrichment $(\mathrm{Zr}, \mathrm{Y}, \mathrm{Nb}$, and REE) exhibited by the rhyolite and the apparently limited role of crystal fractionation, it is probable that the crustal source was enriched. Partial melting of continental crust may be induced by halogen-rich fluids released from underlying basaltic magma, resulting in alkaline rhyolites (Hildreth, 1981; Davies and Macdonald, 1987; Macdonald and others, 1987). Fluorine and chlorine have a stabilizing effect on highly charged cations (Collins and others, 1982) and may have contributed to the enrichment of light REE in the rhyolite by inhibiting fractionation of allanite and monazite (Hildreth, 1979; Crecraft and others, 1981; Ludington, 1981; Miller and Mittlefehldt 1982). Relative depletion of heavy REE has also been attributed to equilibration with residual zircon, (Christiansen and others, 1983), fractionation of hornblende (Frey and others, 1978), partitioning of heavier REE into a volatile-rich fluid (Christiansen and others, 1983), or derivation from a source depleted in heavy REE. The high levels of $\mathrm{Zr}$ and the absence of any hornblende in the rhyolite make the first two processes unlikely. The simplest explanation is that the relative enrichment in light REE as well as the overall enrichment of incompatible elements are the results of an enriched source and the small degrees of partial melting typically associated with alkaline rocks.

Eruptive style.-In many respects, the Wamsutta volcanic suite resembles some of the smaller volcanic centers within continental rift systems. As demonstrated earlier, the Wamsutta suite is geochemically very similar to bimodal volcanics of the Naivasha volcanic field in Kenya (Davies and Macdonald, 1987; Macdonald and others, 1987). The relatively small volume of the Wamsutta suite and the nature of the extrusive and pyroclastic units are reminiscent of extension-related volcanism produced at the Inyo/Mono craters on the flank of Long Valley caldera. Like the Inyo volcanics (Miller, 1985), the Wamsutta volcanics appear to be the result of relatively small eruptive episodes that occurred over a short period of time. The close stratigraphic spacing of 
the six Wamsutta volcanic flows, their parallel trends, and the uneroded surfaces of the basalt flows suggest a rapid succession of events, with insufficient time for extensive sedimentation between eruptions, topographic change, or erosion of the flows. Direct contact between flows of basalt as well as flows of basalt and rhyolite further indicate short time intervals between eruptive episodes. Basalt and rhyolite in direct sharp contact also demonstrate the simultaneous presence of distinct, coeval batches of mafic and felsic magma. Associated sedimentary rocks suggest eruptions took place in an alluvial fan environment with numerous braided streams (Cazier, ms). Such an environment is consistent with the abundant evidence of interaction between magma, water, and mud.

Volcanic activity appears to have initiated with the eruption of high-temperature, low-viscosity magmas producing thin, laterally extensive, possibly pahoehoe-type basalt flows. Local pillow structures and quench textures suggest that portions of the flows were subaqueous. Subsequent rhyolitic flows also appear to have been relatively low-viscosity flows rather than highly viscous dome extrusions, based on the predominance of horizontal flow foliations (absence of ramp structures) and an apparent low aspect ratio. Perlitic textures and low phenocryst abundance indicate that the rhyolitic flows cooled rapidly.

The presence of pyroclastic horizons beneath the capping rhyolite indicates that the passive extrusion of the flow was preceded by an explosive stage. The occurrence of explosive volcanism is consistent with the alkaline nature of the basalt and rhyolite and suggests that the magma sources were rich in volatiles. The explosive and passive phases could represent pre- and post-degassing activity, respectively. This seems probable considering the low vesicularity of the rhyolite. However, given the small scale of these volcanics and the wet environment into which they likely erupted, it is also possible that the explosive event was phreatic.

Implications for tectonism.-Restriction of the Wamsutta rhyolite and basalt to low stratigraphic levels in the Narragansett basin suggests that the volcanics are associated with the early formational stages of the basin. The bimodal and alkaline nature of the Wamsutta suite strengthen this connection, because both features are commonly associated with magmatism occurring in extensional rift environments (Macdonald, 1974b; Ewart, 1979; Metz and Bacon, 1980; Sigurdsson and Sparks, 1981). Although the precise relationship between extension and coeval magma suites is not thoroughly understood, characteristics of the Wamsutta volcanics provide insight into the nature of the rift event that formed the Narragansett basin.

Various tectonic models have been proposed to explain the formation of the Narragansett basin, including pure extension, with no significant strike-slip movement (Cazier, 1987), compression-related wrench faulting (Skehan and others, 1986), and transpressional strike-slip movement (McMaster and others, 1980; Mosher, 1983). A likely origin for the volcanics is a rift or pull-apart event during which deep fracturing of the crust allowed intrusion of basalts from the mantle, resulting in partial melting of crustal rocks and extraction of rhyolitic magmas. Though more detailed speculation on the tectonic setting is difficult, it is apparent that conditions favored rapid passage of compositionally distinct magmas through the crust with minimal differentiation or mixing and ultimately the eruption of mafic and felsic lavas in close spatial and temporal association. These conditions might be favored by an extensional regime marked by brittle failure of the crust (Hildreth, 1981) and/or high rates of extension (Davis and others, 1993).

The source of the tectonic stress driving the basin-forming rift event has not been constrained. A Late Devonian age for the rhyolite indicates that the formation of the Narragansett basin began considerably earlier than previously believed. This raises the possibility of an association between basin formation and the mid-Paleozoic or younger, 
normal ductile shear structures occurring in basement rocks west of the basin (Hamidzada, ms; Hamidzada and others, 1999) and, more generally, an association with the extensional environment that produced alkaline magmatism in the southeastern New England Avalon zone throughout much of the Paleozoic. The Wamsutta suite could represent the culmination of a prolonged phase of anorogenic doming and extension as demonstrated by alkaline plutonic rocks outside the margin of the basin.

A Late Devonian age associates the Wamsutta suite with other Devonian alkaline rocks of southeastern Avalon (Scituate Plutonic Suite, Rattlesnake pluton, Hermes and Zartman, 1992; and several mafic plutons northwest of Boston, Massachusetts, Hepburn and others, 1993) and raises the question of a possible relationship between them. At the least, it appears that this interval is characterized by more igneous activity than previously thought. So far, no convincing connection has been established between the Wamsutta volcanics and any other alkaline rocks in the region. The Wamsutta volcanics are geochemically distinct from alkaline volcanic rocks associated with Rhode Island's Devonian Scituate magma series (Spencer Hill volcanics) and Massachusetts's Late Ordovician Quincy Granite (Blue Hill volcanics) (Hermes and Murray, 1990; Hermes and Ritchie, 1997). However, association with plutonic rocks of the Scituate Igneous Suite has not been definitively ruled out, as there is some compositional overlap between the Wamsutta rhyolite and some varieties of granite.

Comparison with the Canadian Maritimes basin.-The Narragansett basin of southeastern New England and the Canadian Maritimes basin, on strike to the north, share many characteristics, including similar stratigraphy, sedimentology, structure, fossils, and fossil ages, which suggest that they may be counterparts (Mosher and Rast, 1984; Keppie and Dallmeyer, 1989; Muehlberger, 1996). While the bimodal volcanic suite of the Wamsutta Formation appears to be unique in southeastern New England, the Maritimes basin (Magdalen basin to some workers) contains similar DevonianCarboniferous age flows of basalt and rhyolite interbedded with fluvial sedimentary rocks. Associated pyroclastic deposits are also common in both settings, as are features reflecting lava/sediment interaction during extrusion into a wet, sedimentary environment. These similarities suggest the possibility that these basins have related tectonic histories. At the least, it is likely that the bimodal volcanic suites share similar petrogenetic origins.

Magmatism associated with the extensional Maritimes basin ranges in age from Middle-Late Devonian to Middle Pennsylvanian (Pe-Piper and Piper, 1998, fig. 2) and is dominated by gabbro/basalt of tholeiitic character and granites and rhyolites of A-type (Pe-Piper and Piper, 1998; Barr and Peterson, 1998; Dessureau and others, 2000). As in southeastern New England, coeval plutonic rocks are common adjacent to the basin. Southeastern New England also experienced a major Middle-Late Devonian magmatic event, but it produced primarily A-type granites with lesser amounts of mafic rocks. At present, it appears that magmatic activity in and around the Maritimes basin extended to $330 \mathrm{Ma}$ (Dessureau and others, 2000), outlasting igneous activity in southeastern New England. The Devonian age now attributed to the Wamsutta volcanics makes them the youngest alkaline igneous rocks in southeastern New England, with the possible exception of the alkaline granite of northern Rhode Island (Hermes and Zartman, 1985) that yielded a $\mathrm{Pb} / \mathrm{Pb}$ age of about $345 \mathrm{Ma}$ (younger than the Scituate Igneous Suite). However, alkaline igneous activity in southeastern New England began as early as the Late Proterozoic and was repeated during several pre-Devonian episodes through the Ordovician and Silurian (Hermes and Zartman, 1992, and references therein). Representatives of these pre-Devonian alkaline igneous events appear to be absent in the Maritimes.

Compared to the Narragansett basin, volcanic rocks are more abundant, voluminous, and diverse in age in the Maritimes basin. Unlike southeastern New England, 
where felsic compositions (plutonic and extrusive) are most common, extrusive rocks in the Maritimes are predominantly mafic. Nevertheless, bimodal volcanic suites of Devonian-Carboniferous age, similar to the Wamsutta suite, are widespread (Dostal and others, 1983, 1989, 1994; Barr and others, 1985, 1995; Barr and Peterson, 1998; Pe-Piper and Piper, 1998; Piper and others, 1999; Dessureau and others, 2000). Two of the better represented bimodal volcanic units are the Fisset Brook Formation (375 Ma) and the Fountain Lake Group (355-360 Ma) (Barr and others, 1995; Barr and Peterson, 1998; Pe-Piper and Piper, 1998). The Fisset Brook volcanics and the Wamsutta volcanics of the Narragansett basin appear to be the same age.

Most of the mafic rocks in the Maritimes are tholeiites, except for some minor older and younger basalts that are transitional to alkaline, and all exhibit characteristics consistent with continental within-plate origins (Barr and Peterson, 1998; Pe-Piper and Piper, 1998; Dessureau and others, 2000). Wamsutta basalts are notably higher in $\mathrm{TiO}_{2}, \mathrm{P}_{2} \mathrm{O}_{5}$, and alkalies than the tholeiites from eastern Canada. It is possible that the compositional differences partly reflect different source materials in the underlying lithosphere. While the Narragansett basin and Paleozoic alkalic rocks in southeastern New England all occur in the Avalon Zone (fig. 1), the Maritimes basin consists of three different basement terranes - from west to east, the Gander, Avalon, and Meguma Zones (Piper and others, 1999; Dessureau and others, 2000).

The rhyolites in the Maritimes basin, like the Wamsutta rhyolite, are generally similar to A-type granites. The Wamsutta rhyolite resembles the low-Zr rhyolites in eastern Canada (Piper and others, 1999) but has greater abundances of high-fieldstrength elements. The alkaline Spencer Hill and Blue Hills volcanic suites (Hermes and Murray, 1990; Hermes and Ritchie, 1997), with $\mathrm{Zr}$ concentrations greater than $1230 \mathrm{ppm}$, are more similar to the high-Zr rhyolites of eastern Canada (Piper and others, 1999) but have greater abundances of other high-field-strength elements.

The characteristics of the bimodal volcanic suite described here and their newly recognized mid-Paleozoic age contribute to the list of Narragansett basin geologic traits (stratigraphy, sedimentology, and fossil character, et cetera) that more than casually resemble the geology of the Maritime basin in eastern Canada. Both basins share a generally similar assemblage of igneous rock types and ages, and both are thought to have been initiated by crustal thinning and extension. Proposed origins for the Maritimes basin include wrench-type extension, a failed rift above a mantle plume, or post-orogenic collapse (Pe-Piper and others, 1998). Although details on the origins of these basins are unresolved, the characteristics shared by the volcanics of the Maritimes basin and the Narragansett basin suggest that they may have formed by similar processes, and that the two basins may have similar tectonic histories.

\section{CONCLUSIONS}

1. The Wamsutta Formation contains a bimodal suite of volcanic rocks consisting of transitional alkalic basalt flows and rhyolite flows that are geochemically similar to nearby A-type granites. Felsic pyroclastic units with well-preserved relict textures are also present. Basalt and rhyolite in direct contact demonstrate the simultaneous presence of distinct, coeval batches of mafic and felsic magma. Geochemical trends suggest the basalt and rhyolite originated by partial melting of different sources, followed by limited fractional crystallization.

2. Compositionally, the basalt and rhyolite are typical of volcanic rocks originating in an extensional tectonic regime, thus supporting incipient basin formation by rifting or pull-apart processes.

3. The Devonian age of the volcanics and interstratified sedimentary rocks demonstrates that stratigraphically lower units in the Narragansett basin are substan- 
tially older than their previously interpreted Pennsylvanian age and that basin formation occurred much earlier than thought.

4. General similarities between the mid-Paleozoic geology of the southeastern New England Avalon and that in the Maritimes of eastern Canada suggest that the two regions shared similar magmatic/tectonic processes and histories.

\section{ACKNOWLEDGMENTS}

We thank Nasir Hamidzada for his assistance with figure and map preparation, Daniel Murray for helpful discussions throughout the project, and Nancy Nevins for analyzing samples by neutron activation. We are also grateful to Jim Skehan, S.J., Margaret Thompson, and an anonymous reviewer for comments and suggestions that improved this manuscript.

\section{REFERENCES}

Baker, B.H., Goles, G.G., Leeman, W.P., and Lindstrom, M.M., 1977, Geochemistry and petrogenesis of a basalt-benmoreite-trachyte suite from the southern part of the Gregory rift, Kenya: Contributions to Mineralogy and Petrology, v. 64, p. 303-332.

Barberi, F., Santacroce, R., and Varey, J., 1982, Chemical aspects of rift magmatism, in Palmason, G., editor, Continental and oceanic rifts: American Geophysical Union, p. 223-258.

Barr, S.M., Brisebois, D., and Macdonald, A.S., 1985, Carboniferous volcanic rocks of the Magdalen Islands, Gulf of St. Lawrence: Canadian Journal of Earth Sciences, v. 22, p. 1679-1688.

Barr, S.M., Macdonald, A.S., Arnott, A.M., and Dunning, G.R., 1995, Field relations, structure, and geochemistry of the Fisset Brook Formation in the Lake Ainslie - Gillanders Mountain area, central Cape Breton Island, Nova Scotia: Atlantic Geology, v. 31, p. 127-139.

Barr, S.M., and Peterson, K.C.A., 1998, Field relationships and petrology of the Late Devonian Fisset Brook Formation in the Cheticamp area, western Cape Breton Island, Nova Scotia: Atlantic Geology, v. 34, p. 121-132.

Boynton, W.V., 1984, Geochemistry of the rare earth elements: meteorite studies, in Henderson, P., editor, Rare earth element geochemistry: Elsevier, Amsterdam, New York, p. 63-114.

Carr, M.J., 1987, IGPET II Computer Program: New Brusnwick, New Jersey, Rutgers University.

Cazier, E.C., ms, 1984, Late Paleozoic tectonic evolution of the Norfolk basin, Southeastern Massachusetts: M.A. thesis, University of Texas, Austin, 147 p.

1987, Late Paleozoic tectonic evolution of the Norfolk basin, southeastern Massachusetts: Journal of Geology, v. 95, p. 55-73.

Christiansen, E.H., Burt, D.M., Sheridan, M.F., and Wilson, R.T., 1983, The petrogenesis of topaz rhyolites from the western United States: Contributions to Mineralogy and Petrology, v. 83, p. 16-30.

Civetta, L., Cornette, Y., Crisci, G., Gillot, P.Y., Orsi, G., and Requejo, C.S., 1984, Geology, geochronology and chemical evolution of the island of Pantelleria: Geological Magazine, v. 121, no. 6, p. 541-562.

Cleaves, A.B., ms, 1929, Geology of the Wamsutta red beds and their relation to associated sedimentary and igneous rocks in the Narragansett basin: M.A. thesis, Brown University, Providence, Rhode Island.

Cogswell, J.P.M., and Mosher, S., 1994, Late-stage Alleghanian wrenching of the southwestern Narragansett basin, Rhode Island: American Journal of Science, v. 294, p. 861-901.

Collins, W.J., Beams, S.D., White, A.J.R., and Chappell, B.W., 1982, Nature and origin of A-type granites with particular reference to southeastern Australia: Contributions to Mineralogy and Petrology, v. 80, p. 189-200.

Crecraft, H.R., Nash, W.P., and Evans, S.H., 1981, Late Cenozoic volcanism of Twin Peaks, Utah: Geology and Petrology: Journal of Geophysical Research, v. 86, p. 10303-10320.

Davies, G.R., and Macdonald, R., 1987, Crustal influences in the petrogenesis of the Naivasha basalt-rhyolite complex: combined trace element and $\mathrm{Sr}-\mathrm{Nd}-\mathrm{Pb}$ isotope constraints: Journal of Petrology, v. 28, p. $1009-1031$.

Davis, J.M., Elston, W.E., and Hawkesworth, C.J., 1993, Basic and intermediate volcanism of the MogollonDatil volcanic field: implications for mid-Tertiary tectonic transitions in southwestern New Mexico, USA, in Prichard, H.M., Alabaster, T., Harris, N.B.W., and Neary, C.R., editors., Magmatic processes and plate tectonics: Geological Society Special Publication 76, p. 469-488.

Dessureau, G., Piper, D.J.W., and Pe-Piper, G., 2000, Geochemical evolution of earliest Carboniferous continental tholeiitic basalts along a crustal-scale shear zone, southwestern Maritimes basin, eastern Canada: Lithos, v. 50, p. 27-50.

Dostal, J., Keppie, J.D., and Dupuy, C., 1983, Petrology and geochemistry of Devono-Carboniferous volcanic rocks in Nova Scotia: Maritime Sediments and Atlantic Geology, v. 19, p. 59-71.

Dostal, J., Laurent, R., and Keppie, J.D., 1994, Late Silurian-Early Devonian rifting during transpression in the southern Gaspe Peninsula (Quebec): petrogenesis of volcanic rocks: Canadian Journal of Earth Sciences, v. 30, p. 2283-2294.

Dostal, J., Wilson, R.A, and Keppie, J.D., 1989, Geochemistry of Siluro-Devonian Tobique Volcanic belt in northern and central New Brunswick (Canada): tectonic implications: Canadian Journal of Earth Sciences, v. 26, p. 1282-1296. 
Eaton, H.N., 1925, Structural relations of the igneous rocks of the Wamsutta red beds, Massachusetts [abstract]: Geological Society of America Bulletin, v. 36, no. 1, p. 165-166.

Eby, G.N., 1992, Chemical subdivision of the A-type granitoids: petrogenetic and tectonic implications: Geology, v. 20, p. 641-644.

Ewart, A., 1979, A review of the mineralogy and chemistry of Tertiary-Recent dacitic, latitic, rhyolitic, and related salic volcanic rocks, in Barker, F., editor, Trondhjemites, dacites, and related rocks: Amsterdam, Elsevier, p. 13-121.

Floyd, P.A., and Winchester, J.A., 1975, Magma type and tectonic setting discrimination using immobile elements: Earth and Planetary Science Letters, v. 27, p. 211-218.

Frey, F.A., Chappell, B.W., and Roy, S.D., 1978, Fractionation of rare-earth elements in the Tuolumne Intrusive series, Sierra Nevada batholith, California: Geology, v. 6, p. 239-242.

Grew, E.S., and Day, H.W., 1972, Staurolite, kyanite, and sillimanite from the Narragansett basin of Rhode Island: United States Geological Survey Professional Paper 800-D, p. D151-D157.

Gwinn, R., and Hess, P.C., 1989, Iron and titanium solution properties in peraluminous and peralkaline rhyolitic liquids: Contributions to Mineralogy and Petrology, v. 101, p. 326-338.

Hamidzada, N.A., ms, 1988, Petrology and structure of NE Scituate Pluton: M.S. thesis, University of Rhode Island, Kingston, Rhode Island, $294 \mathrm{p}$.

Hamidzada, N.A., Murray, D.P. Hermes, O.D., and Bettez, E.A., 1999, The relation of pre-Alleghanian extensional tectonics to basin formation in Rhode Island: Geological Society of America Abstracts with Program, v. 31, p. 20.

Hepburn, J.C., Hon, R., Dunning, G.R., Bailey, R.H., and Galli, K., 1993, The Avalon and Nashoba terranes (eastern margin of the Appalachian Orogen in southeastern New England), in Cheney, J.T. and Hepburn, J.C., editors, Field Trip Guidebook for the Northeastern United States, Geological Society of America, Boston, 1993: Amherst, Massachusetts, University of Massachusetts, Department of Geology and Geography, v. 2, p. X1-X31.

Hepburn, J.C., and Rehmer, J.A., 1981, The diagenetic to metamorphic transition in the Narragansett and Norfolk basins, Massachusetts and Rhode Island, in Hermes, O.D., and Boothroyd, J.C., editors, Guidebook to Geologic Field Studies in Rhode Island and adjacent areas: New England Intercollegiate Geological Conference, $73^{\text {rd }}$ Annual Meeting, University of Rhode Island, Kingston, Rhode Island, p. $138-143$.

Hermes, O.D., Gromet, L.P., and Murray, D.P. (Compilers), 1994, Bedrock geologic map of Rhode Island: Rhode Island Map Series No. 1., Scale 1:100,000, University of Rhode Island, Kingston, Rhode Island.

Hermes, O.D., and Murray, D.P., 1988, Middle Devonian to Permian plutonism and volcanism in the North American Appalachians, in Harris, A.L. and Fettes, D.J., editors, The Caledonide-Appalachian Orogen: The Geological Society of London, Special Publication 38, p. 559-571.

1990, Geochemical distinctions of Late Proterozoic and Paleozoic volcanism in the Avalon zone of New England, in Socci, A.D., Skehan, J.W., and Smith, G., editors, Geology of the composite Avalon Terrane of southern New England: Geological Society of America Special Paper 245, p. 97-112.

Hermes, O.D., and Ritchie, D., 1997, Application of petrographic and geochemical methods to sourcing felsitic archaeological materials in southeastern New England: Geoarchaeology, v. 12, no. 1, p. $1-30$.

Hermes, O.D., and Zartman, R.E., 1985, Late Precambrian and Devonian plutonic terrane within the Avalon Zone of Rhode Island: Geological Society of America Bulletin, v. 96, p. 272-282.

1992, Late Proterozoic and Silurian alkaline plutons within the southeastern New England Avalon Zone: Journal of Geology, v. 100; 4, p. 477-486.

Hildreth, W., 1979, The Bishop Tuff: Evidence for the origin of compositional zonation in silicic magma chambers: Geologic Society of America Special Paper 180, p. 43-75.

1981, Gradients in silicic magma chambers: Implications for lithospheric magmatism: Journal of Geophysical Research, v. 86, no. B11, p. 10153-10192.

Irvine, T.N., and Baragar, W.R.A., 1971, A guide to the chemical classification of the common volcanic rocks: Canadian Journal of Earth Sciences, v. 8, p. 523-548.

Keppie, J.D., and Dallmeyer, R.D., compilers, 1989, Tectonic map of pre-Mesozoic terranes in circumAtlantic Phanerozoic orogens: International Geological Correlation Programme, Terranes in circumAtlantic Paleozoic orogens, Project \#233.

Knox, A.S., 1944, A Carboniferous flora from the Wamsutta Formation of southeastern Massachusetts: American Journal of Science, v. 242, p. 130-138.

Kushiro, I., 1960, Si-Al relations in clinopyroxenes from igneous rocks: American Journal of Science, v. 258, p. 548-554.

Le Bas, M.J., 1962, The role of aluminum in igneous pyroxenes in relation to their parentage: American Journal of Science, v. 260, p. 267-288.

Le Bas, M.J., Le Maitre, R.W., Streckeisen, A., and Zanettin, B., 1986, A chemical classification of volcanic rocks based on a total alkali-silica diagram: Journal of Petrology, v. 27, 3, p. 745-750.

Leat, P.T., Jackson, S.E., Thorpe, R.S., and Stillman, C.J., 1986, Geochemistry of bimodal basalt-subalkaline/ peralkaline rhyolite provinces within the Southern British Caledonides: Geological Society of London Journal, v. 143, p. 259-273.

Lidback, M.M., ms, 1977, Areal geology of the Attleboro quadrangle Massachusetts-Rhode Island: Ph.D. thesis, Boston University, Boston, Massachusetts.

Ludington, S., 1981, The Redskin Granite: Evidence for thermogravitational diffusion in a Precambrian granite batholith: Journal of Geophysical Research, v. 86, p. 10423-10430. 
Lyons, P.C., ms, 1969, Bedrock geology of the Mansfield quadrangle, Massachusetts: Ph.D. thesis, Boston University, Boston, Massachusetts, 283 p.

1984, Carboniferous megaflora zonation of New England, in Sutherland, P.K., and Manger, W.L., editors, Neuvieme Congres International de Stratigraphie de Carbonifere, Compte Rendu, Biostratigraphy: Carbondale, Illinois, Southern Illinois University Press, v. 2, p. 503-514.

Lyons, P.C., and Chase, H.B., 1976, Coal stratigraphy and flora of the northwestern Narragansett basin, in Cameron, B., editor, A guidebook for field trips to the Boston area and vicinity: New England Intercollegiate Geologic Conference, 68th Annual Meeting, Boston University, Boston, Massachusetts, p. 405-427.

Lyons, P.C., and Darrah, W.C., 1977, Floral evidence for Upper Pennsylvanian in the Narragansett basin, southeastern New England (abstract): Geological Society of America Abstracts with Programs, v. 9, no. 3, p. 297.

Lyons, P.C., Tiffney, B., and Cameron, B.W., 1976, Early Pennsylvanian age of the Norfolk basin, southeastern Massachusetts, based on plant megafossils, in Lyons, P.C., and Brownlow, A., editors, Studies in New England Geology: Geological Society of America Memoir 146, p. 181-197.

Lytton, G.W., ms, 1941, The geologic structure of the igneous and sedimentary rocks of South Attleboro, Massachusetts: M.A. thesis, Brown University, Providence, Rhode Island.

Macdonald, R., 1974a, Nomenclature and petrochemistry of the peralkaline oversaturated extrusive rocks: Bulletin Volcanologique, v. 38, p. 498-516.

1974b, Tectonic settings and magma associations: Bulletin Volcanologique, v. 38, p. 575-593.

Macdonald, R., and Bailey, D.K., 1973, The chemistry of the peralkaline oversaturated obsidians: United States Geological Survey Professional Paper 440-N-1, p. N1-N37.

Macdonald, R., Davies, G.R., Bliss, C.M., Leat, P.T., Bailey, D.K., and Smith, R.L., 1987, Geochemistry of high-silica peralkaline rhyolites, Naivasha, Kenya Rift Valley: Journal of Petrology, v. 28, p. 979-1008.

Maria, A., ms, 1990, Petrology and significance of Carboniferous volcanics, South Attleboro, Massachusetts: M.S. thesis, University of Rhode Island, Kingston, Rhode Island, 172 p.

McMaster, R.L., de Boer, J., and Collins, B.P., 1980, Tectonic development of southern Narragansett Bay and offshore Rhode Island: Geology, v. 8, no. 10, p. 496-500.

Meschede, M., 1986, A method of discriminating between different types of mid-ocean ridge basalts and continental tholeiites with the Nb-Zr-Y diagram: Chemical Geology, v. 56, p. 207-218.

Metz, J., and Bacon, C.R., 1980, Quenched blobs of mafic magma in high-silica rhyolite of the Coso volcanic field, California: Geological Society of America Abstracts with Programs, v. 12, p. 120.

Miller, C.D., 1985, Holocene eruptions at the Inyo volcanic chain, California: implications for possible eruptions in Long Valley caldera: Geology, v. 13, p. 14-17.

Miller, C.F., and Mittlefehldt, D.V., 1982, Depletion of light rare-earth elements in felsic magmas: Geology, v. 10, p. $129-133$.

Miyashiro, A., 1978, Nature of alkalic volcanic rock series: Contributions to Mineralogy and Petrology, v. 66, p. 91-104.

Mosher, S., 1981, Late Paleozoic deformation of the Narragansett basin, Rhode Island: Geological Society of America Abstracts with Programs, v. 13, p. 515.

1983, Kinematic history of the Narragansett basin, Massachusetts and Rhode Island: Constraints on late Paleozoic plate reconstructions: Tectonics, v. 2, no. 4, p. 327-344.

Mosher, S., and Rast, N., 1984, The deformation and metamorphism of Carboniferous rocks in Maritime Canada and New England, in Hutton, D.H.W., and Sanderson, D.J., editors, Variscan tectonics of the North Atlantic region: Geological Society Special Publication 14, (Oxford, Blackwell Scientific Publications), p. 233-243.

Muehlberger, W.R., compiler, 1996, Tectonic map of North America: The American Association of Petroleum Geologists, Tulsa, Oklahoma.

Murray, D.P., 1988, Post-Acadian metamorphism in the Appalachians, in Harris, A.L., and Fettes, D.J., editors, The Caledonian-Appalachian orogen: Geological Society of London Special Publication 38, p. 597-609.

Murray, D.P, Raben, J.D., Lyons, P.J, and Chase, H.B., 1981, The geological setting of coal and carbonaceous material, Narragansett basin, southeastern New England, in Boothroyd, J.C., and Hermes, O.D., editors, Guidebook for field trips in Rhode Island and adjacent areas: New England Intercollegiate Geological Conference, 73rd annual meeting, University of Rhode Island, p. 181-190.

Murray, D.P., and Skehan, J.W., S.J., 1979, A traverse across the eastern margin of the AppalachianCaledonide orogen, southeastern New England, in Skehan, J.W., S.J., and Osberg, P.H., editors, The Caledonides in the U.S.A.: Geological Excursions in the Northeast Appalachians: International Geological Correlation Program (I.G.C.P.) Project 27, Weston, Massachusetts, Weston Observatory, p. 1-35.

Mutch, T.A., 1968, Pennsylvanian nonmarine sediments of the Narragansett basin, Massachusetts-Rhode Island, in Klein, G. deV., editor, Late Paleozoic and Mesozoic continental sedimentation, northeastern North America: Geological Society of America Special Paper 106, P. 177-209.

Noble, D.C., 1970, Loss of sodium from crystallized comendite welded tuffs of the Miocene Grouse Canyon Member of the Belted Range Tuff, Nevada: Geological Society of America Bulletin, v. 81, p. 2677-2687. 1972, Some observations on the Cenozoic volcano-tectonic evolution of the Great Basin, western United States: Earth and Planetary Science Letters, v. 17, p. 142-150.

O’Hara, K.D., and Gromet, L.P., 1985, Two distinct Late Precambrian terranes in southeastern New England and their Late Paleozoic juxtaposition: American Journal of Science, v. 285, p. 673-709.

Pe-Piper, G., and Piper, D.J.W., 1998, Geochemical evolution of Devonian-Carboniferous igneous rocks of the Magdalen basin, eastern Canada: $\mathrm{Pb}$ - and $\mathrm{Nd}$-isotope evidence for mantle and lower crustal sources: Canadian Journal of Earth Sciences, v. 35, p. 201-221. 
Pearce, J.A., 1982, Trace element characteristics of lavas from destructive plate boundaries, in Thorpe, R.S., editor, Andesites: orogenic andesites and related rocks: Chichester, Wiley, p.525-548.

Pearce, J.A., and Cann, J.R., 1973, Tectonic setting of basic volcanic rocks determined using trace element analyses: Earth and Planetary Science Letters, v. 19, p. 290-300.

Pearce, J.A., and Gale, G.H., 1977, Identification of ore-deposition environment from trace-element geochemistry of associated igneous host rocks, in Volcanic processes in ore genesis: Geological Society of London Publication 7, p. 14-24.

Pearce, J.A., Harris, N.B.W., and Tindle, A.G., 1984, Trace element discrimination diagrams for the tectonic interpretation of granitic rocks: Journal of Petrology, v. 25, p. 956-983.

Pearce, J.A., and Norry, M.J., 1979, Petrogenetic implications of Ti, Zr, Y, and Nb variations in volcanic rocks: Contributions to Mineralogy and Petrology, v. 69, p. 33-47.

Pierce, T.A., ms, 1976, Petrology of dolerite-metadolerite dikes of southeastern New England: M.S. thesis, University of Rhode Island, Kingston, Rhode Island.

Piper, D.J.E., Dessureau, G., and Pe-Piper, G., 1999, Occurrence of early Carboniferous high-Zr rhyolites, Cobequid Highlands, Nova Scotia: Temperature effect of a contemporaneous mafic magma: The Canadian Mineralogist, v.37, p. 619-634.

Severson, R.H., ms, 1981, Depositional environments, facies relationships, and coal occurrence in Carboniferous sediments of the Narragansett basin: M.S. thesis, University of Rhode Island, Kingston, Rhode Island, $113 \mathrm{p}$.

Shaler, N.S., Woodworth, J.B., and Foerste, A.F., 1899, Geology of the Narragansett basin: United States Geological Survey Monograph 33, 402 p.

Sigurdsson, H., and Sparks, R.S.J., 1981, Petrology of rhyolitic and mixed magma ejecta from the 1875 eruption of Askja, Iceland: Journal of Petrology, v. 22, p. 41-81.

Skehan, J.W., S.J., and Murray, D.P., 1979, Geology of the Narragansett basin, Southeastern Massachusetts and Rhode Island, in Cameron, B., editor, Carboniferous basins of southeastern New England: International Congress of Carboniferous Stratigraphy and Geology, $9^{\text {th }}$, Field Trip Guidebook no. 5, Falls Church, Virginia, American Geological Institute, p. 7-35.

Skehan, J.W., S.J., Murray, D.P., Hepburn, J.C., Billings, M.P., Lyons, P.C., and Doyle, R.G., 1979, The Mississippian and Pennsylvanian (Carboniferous) systems in the United States - Massachusetts, Rhode Island, and Maine: United States Geological Survey Professional Paper 1110-A, p. 30 p.

Skehan, J.W., S.J., Rast, N., and Mosher, S., 1986, Paleoenvironmental and tectonic controls of sedimentation in coal forming basins of southeastern New England: Geological Society of America Special Paper 210, p. $9-30$.

Sutherland, D.S., 1974, Petrography and mineralogy of the peralkaline silicic rocks: Bulletin Volcanologique, v. 38 , p. 517-547.

Taylor, S.R., and McLennan, S.M., 1985, The continental crust: its composition and evolution: Oxford, England, Blackwell, 312 p.

Thompson, M.D., and Hermes, O.D., 1990, Ash-flow stratigraphy in the Mattapan Volcanic Complex, greater Boston, Massachusetts, in Socci, A.D., Skehan, J.W., and Smith, G.W., editors: Geological Society of America Special Paper 245, p. 85-95.

Thompson, M.D., Hermes, O.D., Martin, M.W., and Bowring, S.A., 1999, Late Devonian rhyolite in the northwestern Narragansett basin: Geological Society of America, Abstracts with Programs, v. 31, no. 2, p. 73.

Thompson, R.N., 1982, Magmatism of the British Tertiary Volcanic Province: Scottish Journal of Geology, v. 18 , p. $49-107$.

Wilkinson,J.F.G., 1974, The mineralogy and petrography of alkali basaltic rocks, in Sorensen, H., editor, The Alkaline Rocks: New York, John Wiley \& Sons, p. 67-95.

Wilson, M., 1989, Igneous petrogenesis: London, Unwin Hyman, 466 p.

Winchester, J.A., and Floyd, P.A., 1977, Geochemical discrimination of different magma series and their differentiation products using immobile elements: Chemical Geology, V. 20, p. 325-343.

Woods, M.D., ms, 1961, The nature, origin and significance of flow layering in a rhyolite flow, Wamsutta Formation, South Attleboro, Massachusetts: M.S. thesis, Brown University, Providence, Rhode Island, $41 \mathrm{p}$.

Zartman, R.E., and Hermes, O.D., 1987, Archean inheritance in zircons from Late Paleozoic granites from the Avalon zone of southeastern New England: an African connection: Earth and Planetary Science Letters, v. 82, p. 305-315.

Zen, E-an, compiler, 1983, Geological map of Massachusetts: United States Geological Survey, 1:25,000 scale, 3 map sheets. 